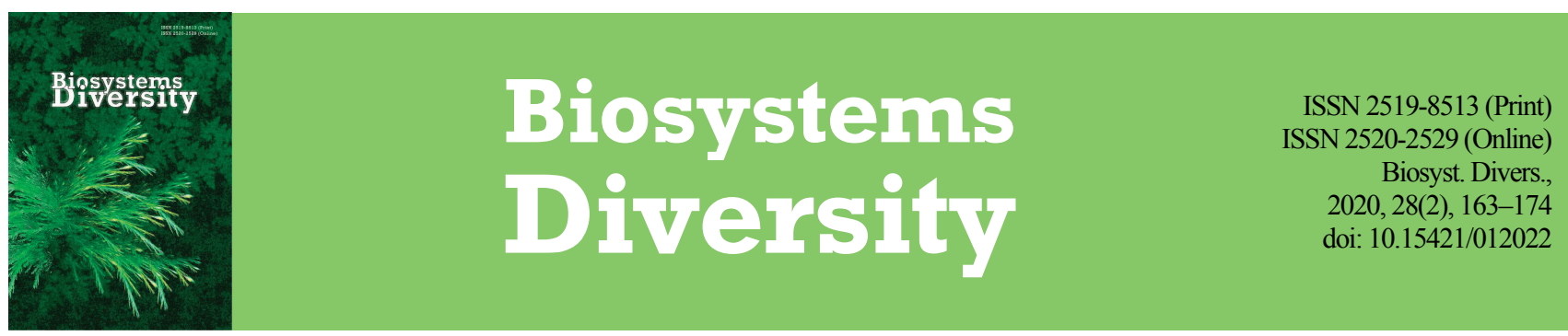

\title{
Ecological-faunistic analysis of ground beetles and tiger beetles (Coleoptera: Carabidae, Cicindelidae) of metropolises of Ukraine
}

\author{
A. V. Putchkov*, V. V. Brygadyrenko**, N. Y. Nikolenko*** \\ *I. I. Schmalhausen Institute of Zoology of National Academy of Sciences of Ukraine, Kyiv, Ukraine \\ **Oles Honchar Dnipro National University, Dnipro, Ukraine \\ ***G. S. Skovoroda Kharkiv National Pedagogical University, Kharkiv, Ukraine
}

Article info

Received 24.03.2020

Received in revised form 26.04.2020

Accepted 27.04.2020

I. I. Schmalhausen Institute of Zoology of National Academy of Sciences of Ukraine,

B. Khmelnytskoho st., 15 , Kyiv, 01030, Ukraine.

Tel.: +38-095-462-62-23.

E-mail:

putchkov@izan.kiev.ua

Oles Honchar Dnipro National University,

Gagarin ave. 72 ,

Dnipro, 49010, Ukraine.

Tel.: +38-050-93-90-788

E-mail:brigad@ua.fm

G. S. Skovoroda

Kharkiv National

Pedagogical University,

Alchevskykh st., 29,

Kharkiv, 61000, Ukraine

$\mathrm{Tel}$ : +38-066-212-13-37.

E-mail:

nikolenkonatala418@

gmaill.com Putchkov, A. V., Brygadyrenko, V. V., \& Nikolenko, N. Y. (2020). Ecological-faunistic analysis of ground beetles and tiger beetles
(Coleoptera: Carabidae, Cicindelidae) of metropolises of Ukraine. Biosystems Diversity, 28(2), 163-174. doi:10.15421/012022

Ground beetles and tiger beetles are the most noticible representatives of predatory invertebrates often used in bioidentificational studies. This article provides quantitative and ecological characteristics of Caraboidea in five metroplises of Ukraine (Dnipro, Donetsk, Kharkiv, Kyiv, and Lviv). In total, in these cities, 237 species of Caraboidea were recorded, belonging to 63 genera and two families - Carabidae (231 species, 61 genera) and Cicindelidae (6 species, 2 genera). By abundance of Carabidae in megapolises, 33 species were identified to abundant. Eudominants were represented by three species: Harpalus rufipes, Pterostichus melanarius and P. oblongopunctatus, dominants - five: Amara aenea, Anchomenus dorsalis, Calathus fuscipes, Harpalus distinguendus and Poecilus versicolor. Subdominants were 25 species: Amara similata, Asaphidion flavipes, Badister bullatus, Bembidion lampros, B. properans, Broscus cephalotes, Calathus ambiguus, C. melanocephalus, Carabus cancellatus, C. coriaceus, C. granulatus, Cylindera germanica, Harpalus affinis, H. anxius, H. griseus, H. latus, H. tardus, Limodromus assimilis, Microlestes minutulus, Nebria brevicollis, Notiophilus palustris, Poecilus cupreus, Pterostichus niger, P. ovoideus and P. strenuus. In the studied cities, 53 species are rare, 151 species were identified to the group of accidental species. For separate cities, number of eudominants ranged from 4 (Donetsk, Kyiv) to 6-8 (Kharkiv, Lviv). Ten species discovered: Amara famelica, A. majuscula, Anisodactylus nemorivagus, Asaphidion pallipes, Badister lacertosus, Blemus discus, Harpalus laeviceps, Limodromus krynickii, Pterostichus minor and Tachyta nana were new for the Steppe zone of Ukraine. Chlaenius aeneocephalus and Brachinus brevicollis were recorded for the first time in the Right Bank of the northern subzone of the steppe zone, and Stenolophus abdominalis mentioned earlier for the far south of Ukraine was for the first time reported for the northern Steppe. Microlestes negrita was new for the Forest Steppe, and Masoreus wetterhalli and Syntomus foveatus, known earlier for the forest zone and the south of the steppe zone, were for the first time reported for its northern subzone. The levels of faunistic similarity of Caraboidea for different metropolises ranged within $0.20-0.60$ for all species and 0.32-0.90 for abundant species. Most similar were carabidofaunas of Kharkiv and Dnipro (similarity index of Jaccard equaled 0.58-0.87), slightly lower in Dnipro and Kyiv $(0.50-0.72)$. Lowest parameters were seen for Lviv compared with the other cities $(0.20-0.32)$. According to the species composition, the most numerous were meadow (119) and forest elements (59 species). To the polytopic group, 25 species are identified, 23 to the steppe group, and 11 species to the litoral group. In numbers in all the metropolises, the polytopic group dominated, among which abundant species comprised almost half. Within the forest group in Dnipro, Donetsk and Kharkiv, 4-6 abundant species were recorded, whereas in Kyiv and Lviv their number was 14-17 species. Within the meadow group, common species were represented by 4-11 species, and in the steppe and litoral groups such species were absent. In relation to moisture, eudominants were mesophiles ( 165 species), including 31 abundant species. According to the trophic specialization, zoophages dominated (146 species), including 24 abundant species. Among zoophytophages, 60 species (seven abundant) were recorded. Phytozoophages were represented by 30 species (two abundant). According to mechanical composition of soil, the more diverse groups were observed to be made up species preferring loamy and clay-loamy soils ( 85 species each), but to the abundant species from these groups, 6 and 10 species respectively were identified. The group of ground beetles with non-manifested preferences to mechanical composition of soil was represented by 41 species, though by number of abundant ( 17 species), the group was dominant. To inhabitants of heavy loamy, sandy and sabulous soils, 4,6 and 16 species correspondingly were identified, and no abundant species were observed. At the level of abundant species, the ecological structure was more similar than shown by the analysis of all the species of ground beetles. Hypothetically, typical representatives of carabid beetles of Ukrainian metropolises could be considered polytopic or meadow (to a lesser extent forest) mesophile zoophages or zoophytophages which prefer loam-clayey soils without preference to one or the other type of soil.

Keywords: Caraboidea; species composition; ecological structure; urban cenoses; cities.

\section{Introduction}

Cities, especially large ones (metropolises) are a quite specific environment for many insects which inhabit original anthropogenic habitats urbocenoses, which according to a number of parameters significantly differ from natural biotopes. The survey of the entomofauna of urbocenoses allows us to understand the peculiarities of its formation, role and adaptive abilities of insects in the conditions of an urban environment, and also perform their ecological and bioidentificational evaluation (Sus- tek, 1987; Magura et al., 2008; Komlyk \& Brygadyrenko, 2019, 2020). Among insects, within the largest order of beetles (Coleoptera), one of the dominating groups in the terrestrial ecosystems is caraboid beetles (ground beetles - Carabidae and tiger beetles - Cicindelidae). Ground beetles as inhabitants of almost all natural and transformed ecosystems in the conditions of urban landscapes were the objective of numerous publications. They included analyses of a number of aspects of the ecological-faunistic structure of the communities, distribution of species of Carabidae in different types of urban landscapes in many cities of a 
number of European countries: Poland (Czechowski, 1981; Kosewska et al., 2013), Germany (Klausnitzer, 1983, 1990; Franzen, 1992); Slovakia (Šustek, 1987), Czech Republic (Hurka \& Jedlickova, 1990), Belarus (Aleksandrowicz, 1997, 2014; Ryzhaya, 2005; Halinovski \& Krytskaya, 2016), Hungary (Magura et al., 2004), Bulgaria (Penev et al., 2008), Finland (Niemelä \& Kotze, 2009) and Spain (Šustek, 2012). These studies resulted in revealing interesting faunistic data and evaluating the role and possibilities of maintaining the representatives of Caraboidea in the conditions of cities (but mostly in city parks). Only some studies focused on comparative analysis of ground beetles in urbocenoses of various geographically remote cities (Balkenhol et al., 1991; Avtaeva et al., 2019).

In Ukraine, such faunistic studies (to different extents) were also sporadically conducted in a number of metropolises - Kyiv (Putchkov et al., 2003; Kirichenko \& Danylkiv, 2011), Kharkiv (Putchkov et al., 2016, 2017b; Komaromi et al., 2018; Nikolenko, 2018), Dnipro (Brygadyrenko, 2015a, 2015b, 2016; Brygadyrenko \& Korolev, 2015) and Lviv (Rizun \& Khrapov, 2001; Rizun \& Dedus', 2016). At the same time, ground beetles were found to be the most studied group, unlike some other families of beetles less studied in transformed biocenoses, for example scarabs Scarabaeidae (Putchkov et al., 2017a), staphilinids Staphilinidae (Putchkov et al., 2020) clown beetles Histeridae (Putchkov \& Komaromi, 2018) and snout beetles Curculionidae (Komaromi et al., 2019; Nazarenko et al., 2020).

However, in each of the abovementioned studies, there are individual data on Carabidae regarding one particular city or even its separate territories (for example, only green plantations). At the same time, to understand the peculiarities of the formation of urban carabidofauna (similarly to other groups of insects) more fully, generalized comparative analysis of its structure should be performed in different, similar by parameters, but geographically remote, metropolises. Such analysis would allow us to obtain more detailed information about the patterns of the reaction of insects to different conditions of their habitat. For ground beetles, such comparisons were performed only on the example of tribes Carabini and Pterostichini (Putchkov et al., 2016, 2017b), whereas for other coleopterans (Scarabaeidae, Staphilinidae, and Curculionidae) only preliminary comparative data for separate cities were obtained (Putchkov et al., 2017, 2020; Nazarenko et al., 2020). The available data suggest greater differences regarding the compositions of species than ecological structure of the community of one or the other group of beetles in metropolises located in different geographic zones.

The objective of this study was to compare the taxonomic composition, abundance of species and the main indicators of the ecological structure of the communities of ground beetles in five metropolises of Ukraine. On the basis of these data, ecological-faunistic analysis should be performed in order to determine the peculiarities of the formation of the communities of Caraboidea in metropolises located in different geographical zones.

\section{Material and methods}

The beetles were counted during more than 20 years - in 19982019 (with separate breaks for some years) in the main urbocenoses of four metropolises of Ukraine: Dnipro, Donetsk, Kharkiv and Kyiv. Moreover, additionally, we used all the available literature on ground beetles in Kyiv (Kirichenko \& Danylkiv, 2011; Kirichenko et al., 2019) and Kharkiv (Dekhtyareva, 2002, 2004). The materials on Donetsk were kindly provided to the authors by V. V. Martynov (Donetsk). The data for Lviv (exclusively the parks) are given on the basis of the literature data (Rizun \& Khrapov, 2001; Rizun \& Dedus', 2016).

In Dnipro, the studies were undertaken in 1998-2019 in urbocenoses, among which the main were also the recreation zones in the Right Bank (Lazar Globa Park, Taras Shevchenko Park, Monastyrsky Island, Sevastopolsky Park, Yury Gagarin Park, Botanical Garden of Dnipro University, Volodia Dubinin Park, Park of the 40th Anniversary of the Liberation of Dnipropetrovsk, Tonnelna Ravine Tract, forest communities of the Zaporizke Highway, river bank communities on the Pobeda Enbankment, Kliuev Garden Square, forest plantations west of the Kievskaia Street, Memory and Reconciliation Park, forest plantations in the areas of Parus residential zone and Diivka), and the areas on the Left Bank of the Dnipro River (Forest-Park of Friendship of Nations, bank communities of the Kuriache Lake, forest plantations west of the Donetske Highway). Except for the recreation zones, pit-fall traps were installed in the yards of private households, alongside highways, near large industrial enterprises (Brygadyrenko \& Reshetniak, 2014a, 2014b, 2016; Korolev \& Brygadyrenko, 2014; Reshetniak et al., 2017).

In Donetsk, ground beetles were collected in 1999-2004 in three city parks: Shcherbakov Park, Lenin Komsomol Park, Putilovsky Park, and also in the Rakovska Ravine (outskirts of the city). In the territory of Kharkiv, the counts were made in 2015-2019 in three parks (Pobeda, Karpovsky Sad, Mashynostroitelei); outskirts of Kharkiv Forest Park in the territory of the city; territories of G. N. Vysotsky Ukrainian Research Institute of Forestry and Forest Melioration (center of the city) and separate yards of the suburban zone (Oleksiivka). A complete characteristic of these urbocenoses was provided in a number of studies on insects of Kharkiv (Komaromi et al., 2018). In Kyiv (the period of 1998-1999, 2002, 2009, 2013), sporadic studies were performed in the main park plantations of city: Lysa Hora Tract (Right Bank of the Lybid River, near the place where it falls into the Dnipro River); Pobeda Park (Left Bank, Darnytsia), Rylsky Park (Right Bank, Holosiivo) and Saint Volodymyr Hill (center), and also separate plantations in Darnytsky and Dniprovsky Districts (Left Bank). The data on tracts of Teremky, Feofaniya and limited territory of Holosiivsky National Park adjacent to the city outskirts (west and south-west part of the city) is taken from the literature sources (see above). The data on Lviv are given exclusively on the basis of the literature sources for two city parks: Sykhivskyi Park and Vinnikovsky (Rizun \& Khrapov, 2001; Rizun \& Diedus, 2016), studies on which lasted for only two years -2001 and 2015.

Thus, the main studies (and analysis of the literature sources) in urbocenoses were conducted in different tree, shrub and decorative plantations of the abovementioned cities. Due to this fact, during the analysis of the material, we did not take into account the carabid fauna of the aquatic and semiaquatic biotopes (in order to prevent confusion with the structure of the communities of ground beetles) which is well represented (and quite specific due to the large amount of water bodies) in Dnipro and Kyiv, but less in Kharkiv and almost absent in Lviv and Donetsk.

During the studies, we used mainly Barber pitfall traps (plastic cups filled with $10 \%$ solution of acetic acid or the so-called live traps without fixator). In each biotope, we installed 10-20 traps in line. The extraction of beetles was made with the intervals of 5-10 days from late April to early October, but sometimes only from May to mid July. In addition, the beetles were recorded and collected on the itineraries in the city, both in the abovementioned areas and others (on lawns and pavements).

In total, in urbocenoses of all the metropolises, over 70,000 specimens of ground beetles were recorded (around 3,500 for Kharkiv, 4,000 for Kyiv, 25,500 for Donetsk, 35,000 for Dnipro, around 2,800 specimens for Lviv). Based on the total number of the selection of no less than 2,500 specimens, according to level of the abundance, five groups were distinguished and presented using a points scale (Table 1): 1 - occasional species or subrecedents (scattered individuals were seen in separate years numbering from one to five specimens for the entire period of the collections, and their share usually equaled less than $0.15 \%$ ); 2 - rare species or recedents (occured sporadically in small numbers, $0.15-0.65 \%$ of total collections); 3 - infrequent species or subdominants (often and continuously occurred in the most biotopes, $0.65-2.49 \%$ of overall collections); 4 - common species or dominants (quite often recorded in most habitats, $2.50-9.99 \%$ of the overall collections), 5 - abundant species (eudominants), recorded in large numbers in all urbocenoses (over 10\% of the overall collections in a metropolis).

In smaller selections (for separate urbocenoses where the total number of the beetles caught was 200-1,000 specimens), the species the share of which exceeded $15 \%$ of the total number of beetles on the plot were identified to eudominants, $5.0-14.9 \%$ to dominant, $1.0-4.9 \%$ to subdominants, $0.30-0.99$ to rare, and less than $0.30 \%$ to occasional species (Putchkov, 2018).

According to the general number scale, we distinguished species the abundance of which is expressed as the ratio of the total of points for all of the metropolises where the species was recorded to the total num- 
ber of cities. Thus, the species identified to eudominants were characterized by the average value of abundance equaling $4.0-5.0$, dominants $3.0-3.9$, subdominants $-1.8-2.9$, recedents $-1.0-1.7$, subrecedents $0.2-0.9$ points. The first three groups (regardless of the amount of the selection) are considered in the study as abundant species for one or the other metropolis. It has to be noted that the data on abundance presented in this article to a sufficient degree reflect the level of overall number of abundant species for the whole period of the surveys taking into consideration most of the urbocenoses. During further studies, some changes may occur in the taxonomic composition and ratio of their number, especially due to recording other rare and occasional species.

To evaluate the similarity of the species composition of separate cities, we used Jaccard coefficients of species similarity. The classification of ecological groups (taking into account the characteristics of imagoes and larvae) is given based on our observations and using the literature data (Sharova, 1981; Putchkov, 2018; Brygadyrenko, 2015a, 2015b, 2015c, 2016). Such divisions into groups are partly subjective, but are provided corresponding to the conditions and pattern of one or the other plot, confinement of species (both imago and larva) to the natural biotopes taking into account the peculiarities of change of the habitat of species in different geographic regions of Ukraine. Some more accurate determinations of biotopic confinement of abundant species of the transitional groups (for example forest- or meadow-marsh, meadow-steppe, forest-shrub and other) are absent, but are briefly considered in the text in the discussion of results. According to preferendum to soils with different mechanical composition, the groups were distinguished with consideration of abundance of larvae, and in case of absence of such data - according to the quantitative characteristics of imago in the areas with different edaphic conditions.

Classification of taxa is given according to the catalogue of beetles of Adephaga suborder of Palearctic (Catalogue of Palearctic Coleoptera..., 2017), and alphabetically within genera and the text (Table 1).
Statistical analysis of the data was performed in Statistica 8.0 software (Statsoft Inc., USA).

\section{Results}

In total, in the urbocenoses of the metropolises (according to our observations and the literature), 237 species of Caraboidea have been recorded (Table 1), belonging to 63 genera and two families - Carabidae (231 species, 61 genera) and Cicindelidae (6 species, 2 genera), which is almost one third of all taxonomic composition of the Caraboidea superfamily in Ukraine. We can confidently presume that the list of ground beetles of urbocenoses (especially for the most poorly studied carabidofauna of Lviv), and other cities of Ukraine, where such studies have not been conducted, will increase to 250-260 species (mainly due to rare species).

Taxonomically the richest genera were Harpalus (34), Amara (26) and Carabus (22 species). Quite diverse were genera Pterostichus (16), Bembidion (9), Ophonus (8), Poecilus (7), Badister (6), Agonum, Calathus, Notiophilus and Stenolophus (5 species each). The remaining 50 genera were represented by $1-4$ species (Table 1 ).

Taxonomic diversity of ground beetles to one extent or the other is specific. In urbocenoses of Dnipro, 156 species have been recorded, 119 in Donetsk, 125 in Kharkiv, 137 in Kyiv, and only 66 in Lviv. The latter circumstance is due to the short period of the studies (only two years) and the lowest number of urbocenoses taken into consideration (only two parks). At the same time, the number of species and the ratio of quantitatively different groups of ground beetles (abundant, rare, and occasional) differed to different extent. For some cities (except Lviv), the number of species of the abundant groups ranged from 19 (Donetsk) to 33 species (Kyiv), in percentage accounting for 14.1\% (Dnipro) to $24.1 \%$ (Kyiv). The number of recedent species and subrecedents did not differ much for most of the cities (Table 1).

\section{Table 1}

Faunistic and ecological characteristics of beetles of Cicindelidae and Carabidae families in metropolises of Ukraine

\begin{tabular}{|c|c|c|c|c|c|c|c|c|c|c|c|}
\hline No & Species & Dnipro & Donetsk & Kharkiv & Kyiv & Lviv & $\begin{array}{l}\text { Total for } \\
\text { five cities }\end{array}$ & $\begin{array}{l}\text { Biotopic } \\
\text { preferred } \\
\text { range }\end{array}$ & $\begin{array}{l}\text { Humidi- } \\
\text { fication } \\
\text { preferred } \\
\text { range }\end{array}$ & $\begin{array}{l}\text { Trophic } \\
\text { characte- } \\
\text { rization }\end{array}$ & $\begin{array}{c}\text { Soil } \\
\text { texture } \\
\text { preferred } \\
\text { range } \\
\end{array}$ \\
\hline \multicolumn{12}{|c|}{ Cicindelidae } \\
\hline 1 & Cicindela campestris Linnaeus, 1758 & 2 & 1 & 1 & 2 & 0 & 1.2 & md & $\mathrm{mz}$ & zo & $\mathrm{cm}$ \\
\hline 2 & C. hybrida Linnaeus, 1758 & 0 & 0 & 1 & 2 & 0 & 0.6 & $\mathrm{pt}$ & $\mathrm{mz}$ & zo & sd \\
\hline 3 & C. maritima kirgisica Mandl, 1936 & 2 & 0 & 0 & 0 & 0 & 0.4 & lt & $\mathrm{mh}$ & zo & sd \\
\hline 4 & C. soluta Dejean, 1822 & 2 & 0 & 0 & 1 & 0 & 0.6 & $\mathrm{fr}$ & $\mathrm{mz}$ & zo & $\mathrm{sd}$ \\
\hline 5 & Cylindera arenaria viennensis (Schrank, 1781) & 1 & 0 & 0 & 1 & 0 & 0.4 & md & $\mathrm{mz}$ & zo & sd \\
\hline 6 & C. germanica (Linnaeus, 1758) & 2 & 2 & 3 & 2 & 1 & 2.0 & md & $\mathrm{mz}$ & zo & $\mathrm{cm}$ \\
\hline \multicolumn{12}{|c|}{ Carabidae } \\
\hline 7 & Leistus ferrugineus (Linnaeus, 1758) & 1 & 3 & 1 & 1 & 0 & 1.2 & fr & $\mathrm{mz}$ & zo & $\operatorname{lm}$ \\
\hline 8 & L. piceus (Frölich, 1799) & 0 & 0 & 0 & 0 & 3 & 0.6 & fr & $\mathrm{mz}$ & zo & $\operatorname{lm}$ \\
\hline 9 & L. rufomarginatus (Duftschmid, 1812) & 0 & 0 & 0 & 0 & 1 & 0.2 & fr & $\mathrm{mh}$ & zo & $\operatorname{lm}$ \\
\hline 10 & Nebria brevicollis (Fabricius, 1792) & 0 & 0 & 0 & 4 & 5 & 1.8 & fr & $\mathrm{mh}$ & zo & $\operatorname{lm}$ \\
\hline 11 & Notiophilus aquaticus (Linnaeus, 1758) & 0 & 0 & 1 & 0 & 0 & 0.2 & md & $\mathrm{mh}$ & zo & $\mathrm{cm}$ \\
\hline 12 & N. biguttatus (Fabricius, 1779) & 1 & 2 & 2 & 2 & 1 & 1.6 & fr & $\mathrm{mz}$ & zo & $\mathrm{cm}$ \\
\hline 13 & N. germinyi (Fauvel in: Grenier, 1863)* & 0 & 0 & 2 & 1 & 0 & 0.6 & md & $\mathrm{mh}$ & zo & $\mathrm{cm}$ \\
\hline 14 & N. laticollis Chaudoir, 1850 & 3 & 1 & 3 & 1 & 0 & 1.6 & md & $\mathrm{mz}$ & zo & $\mathrm{cm}$ \\
\hline 15 & N. palustris (Duftschmid, 1812) & 1 & 4 & 1 & 2 & 1 & 1.8 & fr & $\mathrm{mz}$ & zo & $\mathrm{cm}$ \\
\hline 16 & Calosoma auropunctatum (Herbst, 1784) & 2 & 1 & 2 & 0 & 0 & 1.0 & md & $\mathrm{mz}$ & zo & $\mathrm{cm}$ \\
\hline 17 & C. denticolle Gebler, 1833 & 1 & 1 & 0 & 0 & 0 & 0.4 & md & $\mathrm{mz}$ & zo & $\mathrm{cm}$ \\
\hline 18 & C. inquisitor (Linnaeus, 1758) & 1 & 3 & 3 & 1 & 0 & 1.6 & $\mathrm{fr}$ & $\mathrm{mz}$ & zo & df \\
\hline 19 & Carabus arvensis Herbst, 1784 & 0 & 0 & 0 & 3 & 4 & 1.4 & fr & $\mathrm{mz}$ & zo & $\mathrm{cm}$ \\
\hline 20 & C. besseri (Fischer von Waldheim, 1822 & 1 & 0 & 0 & 0 & 0 & 0.2 & md & $\mathrm{mz}$ & zo & $\mathrm{cl}$ \\
\hline 21 & C. cancellatus Illiger, 1798 & 1 & 1 & 3 & 4 & 0 & 1.8 & $\mathrm{pt}$ & $\mathrm{mz}$ & zo & df \\
\hline 22 & C. clathratus Linnaeus, 1761 & 1 & 0 & 1 & 0 & 0 & 0.4 & md & $\mathrm{mh}$ & zo & $\mathrm{cm}$ \\
\hline 23 & C. convexus Fabricius, 1775 & 1 & 3 & 0 & 4 & 0 & 1.6 & fr & $\mathrm{mz}$ & zo & $\mathrm{cm}$ \\
\hline 24 & C. coriaceus Linnaeus, 1758 & 0 & 0 & 0 & 4 & 5 & 1.8 & fr & $\mathrm{mz}$ & zo & $\mathrm{cm}$ \\
\hline 25 & C. estreicheri Fischer von Waldheim, 1822 & 1 & 1 & 1 & 0 & 0 & 0.6 & $\mathrm{md}$ & $\mathrm{mz}$ & zo & $\mathrm{cm}$ \\
\hline 26 & C. excellens Fabricius, 1798 & 1 & 0 & 0 & 4 & 0 & 1.0 & $\mathrm{md}$ & $\mathrm{mz}$ & zo & $\operatorname{lm}$ \\
\hline 27 & C. glabratus Paykull, 1790 & 0 & 0 & 1 & 2 & 5 & 1.6 & fr & $\mathrm{mz}$ & zo & $\mathrm{cm}$ \\
\hline 28 & C. granulatus Linnaeus, 1758 & 2 & 2 & 3 & 4 & 1 & 2.4 & $\mathrm{pt}$ & $\mathrm{mz}$ & zo & df \\
\hline 29 & C. hortensis Linnaeus, 1758 & 0 & 0 & 0 & 1 & 0 & 0.2 & fr & $\mathrm{mz}$ & zo & $\mathrm{cm}$ \\
\hline 30 & C. hungaricus scythus Motschulsky, 1847 & 1 & 1 & 0 & 0 & 0 & 0.4 & st & $\mathrm{mx}$ & zo & $\mathrm{cl}$ \\
\hline 31 & C. intricatus Linnaeus, 1761 & 0 & 0 & 0 & 0 & 1 & 0.2 & fr & $\mathrm{mz}$ & zo & $\mathrm{cm}$ \\
\hline 32 & C. linnaei Panzer, 1810 & 0 & 0 & 0 & 0 & 2 & 0.4 & fr & $\mathrm{mz}$ & zo & $\mathrm{cm}$ \\
\hline
\end{tabular}




\begin{tabular}{|c|c|c|c|c|c|c|c|c|c|c|c|}
\hline No & Species & Dnipro & Donetsk & Kharkiv & Kyiv & Lviv & $\begin{array}{l}\text { Total for } \\
\text { five cities }\end{array}$ & $\begin{array}{c}\text { Biotopic } \\
\text { preferred } \\
\text { range }\end{array}$ & $\begin{array}{l}\text { Humidi- } \\
\text { fication } \\
\text { preferred } \\
\text { range } \\
\end{array}$ & $\begin{array}{c}\text { Trophic } \\
\text { characte- } \\
\text { rization }\end{array}$ & $\begin{array}{c}\text { Soil } \\
\text { texture } \\
\text { preferred } \\
\text { range } \\
\end{array}$ \\
\hline 33 & C. marginalis Fabricius, 1794 & 1 & 2 & 3 & 0 & 0 & 1.2 & fr & $\mathrm{mz}$ & zo & $\mathrm{cm}$ \\
\hline 34 & C. menetriesi Hummel, 1827 & 0 & 0 & 0 & 1 & 0 & 0.2 & fr & $\mathrm{hm}$ & zo & $\operatorname{lm}$ \\
\hline 35 & C. nemoralis O.Müller, 1764) & 0 & 0 & 5 & 3 & 0 & 1.6 & fr & $\mathrm{mz}$ & zo & $\operatorname{lm}$ \\
\hline 36 & a C. perrini Dejean, 1831 & 0 & 1 & 0 & 0 & 0 & 0.2 & st & $\mathrm{mx}$ & zo & $\mathrm{cm}$ \\
\hline 361 & b C. scabriusculus Olivier, 1795 & 2 & 0 & 0 & 2 & 0 & 0.8 & md & $\mathrm{mz}$ & zo & $\mathrm{cm}$ \\
\hline 37 & C. sibiricus errans Fischer von Waldheim, 1823 & 0 & 2 & 0 & 0 & 0 & 0.4 & $\mathrm{md}$ & $\mathrm{mz}$ & zo & $\mathrm{cl}$ \\
\hline 38 & C. sibiricus fossularius Obydov, 2007 & 1 & 0 & 0 & 0 & 0 & 0.2 & md & $\mathrm{mz}$ & zo & $\mathrm{cm}$ \\
\hline 39 & C. variolosus Fabricius, 1787 & 0 & 0 & 0 & 0 & 1 & 0.2 & fr & $\mathrm{hm}$ & zo & $\operatorname{lm}$ \\
\hline 40 & C. violaceus Linnaeus, 1758 & 0 & 0 & 0 & 1 & 1 & 0.4 & fr & $\mathrm{mz}$ & zo & $\operatorname{lm}$ \\
\hline 41 & Cychrus caraboides (Linnaeus, 1758) & 0 & 0 & 0 & 1 & 3 & 0.8 & fr & $\mathrm{mz}$ & zo & $\operatorname{lm}$ \\
\hline 42 & Blethisa multipunctata (Linnaeus, 1758) & 1 & 0 & 0 & 0 & 0 & 0.2 & md & $\mathrm{mh}$ & zo & $\operatorname{lm}$ \\
\hline 43 & Elaphrus uliginosus (Fabricius, 1775)* & 1 & 0 & 0 & 0 & 0 & 0.2 & lt & $\mathrm{hm}$ & zo & $\operatorname{lm}$ \\
\hline 44 & Loricera pilicornis (Fabricius, 1775) & 1 & 0 & 1 & 1 & 1 & 0.8 & md & $\mathrm{mh}$ & zo & $\operatorname{lm}$ \\
\hline 45 & Scarites terricola Bonelli, 1813* & 1 & 0 & 0 & 0 & 0 & 0.2 & st & $\mathrm{mz}$ & zo & $\mathrm{cm}$ \\
\hline 46 & Clivina collaris (Herbst, 1784)* & 0 & 1 & 1 & 1 & 1 & 0.8 & md & $\mathrm{mh}$ & zo & $\mathrm{cm}$ \\
\hline 47 & C. fossor (Linnaeus, 1758)* & 2 & 1 & 2 & 1 & 0 & 1.2 & $\mathrm{pt}$ & $\mathrm{mz}$ & $\mathrm{zf}$ & df \\
\hline 48 & Dyschiriodes globosus Herbst, 1783 & 1 & 0 & 1 & 0 & 1 & 0.6 & md & $\mathrm{mz}$ & zo & df \\
\hline 49 & Broscus cephalotes (Linnaeus, 1758) & 3 & 1 & 3 & 3 & 0 & 2.0 & $\mathrm{md}$ & $\mathrm{mz}$ & zo & $\mathrm{df}$ \\
\hline 50 & B. semistriatus (Dejean, 1828) & 1 & 1 & 0 & 0 & 0 & 0.4 & md & $\mathrm{mz}$ & zo & $\mathrm{cm}$ \\
\hline 51 & Blemus discus (Fabricius, 1792) & 1 & 0 & 0 & 0 & 1 & 0.4 & $\mathrm{md}$ & $\mathrm{mz}$ & zo & $\mathrm{cm}$ \\
\hline 52 & Trechus quadristriatus (Schrank, 1781) & 1 & 0 & 1 & 2 & 1 & 1.0 & $\mathrm{pt}$ & $\mathrm{mz}$ & zo & df \\
\hline 53 & T. secalis (Paykull, 1790) & 0 & 0 & 0 & 0 & 2 & 0.4 & fr & $\mathrm{mz}$ & zo & $\operatorname{lm}$ \\
\hline 54 & Tachys scutellaris (Stephens, 1829) & 1 & 0 & 0 & 0 & 0 & 0.2 & lt & $\mathrm{mh}$ & zo & $\mathrm{cl}$ \\
\hline 55 & Tachyta nana (Gyllenhal, 1810) & 1 & 1 & 1 & 2 & 0 & 1.0 & fr & $\mathrm{mz}$ & zo & df \\
\hline 56 & Asaphidion flavipes (Linnaeus, 1761) & 2 & 2 & 3 & 2 & 0 & 1.8 & md & $\mathrm{mz}$ & zo & $\mathrm{cm}$ \\
\hline 57 & A. pallipes (Duftschmid, 1812) & 2 & 1 & 2 & 2 & 0 & 1.4 & $\mathrm{md}$ & $\mathrm{mh}$ & zo & $\mathrm{cm}$ \\
\hline 58 & Bembidion assimile Gyllenhal, 1810 & 0 & 1 & 1 & 0 & 0 & 0.4 & $\mathrm{md}$ & $\mathrm{mh}$ & zo & $\mathrm{cm}$ \\
\hline 59 & B. biguttatum (Fabricius, 1779) & 2 & 0 & 1 & 1 & 1 & 1.0 & md & $\mathrm{mz}$ & zo & $\mathrm{cm}$ \\
\hline 60 & B. lampros (Herbst, 1784) & 4 & 1 & 1 & 1 & 2 & 1.8 & $\mathrm{md}$ & $\mathrm{mz}$ & zo & $\mathrm{cm}$ \\
\hline 61 & B. nerescheimeri J. Müller, 1929 & 0 & 0 & 0 & 0 & 3 & 0.6 & md & $\mathrm{mh}$ & zo & $\mathrm{cm}$ \\
\hline 62 & B. properans (Stephens, 1829) & 2 & 2 & 2 & 2 & 1 & 1.8 & $\mathrm{pt}$ & $\mathrm{mz}$ & zo & df \\
\hline 63 & B. quadrimaculatus (Linnaeus, 1761) & 0 & 1 & 1 & 1 & 0 & 0.6 & md & $\mathrm{mz}$ & zo & $\mathrm{cm}$ \\
\hline 64 & B. tetracolum Say, 1823 & 0 & 0 & 0 & 1 & 1 & 0.4 & $\mathrm{md}$ & $\mathrm{mz}$ & zo & $\mathrm{sl}$ \\
\hline 65 & B. stomoides (Dejean, 1831) & 0 & 0 & 0 & 0 & 3 & 0.6 & $\mathrm{fr}$ & $\mathrm{mz}$ & zo & $\mathrm{cm}$ \\
\hline 66 & B. varium (Olivier, 1795) & 2 & 0 & 1 & 2 & 0 & 1.0 & lt & $\mathrm{mh}$ & zo & $\mathrm{sl}$ \\
\hline 67 & Patrobus atrorufus (Ström, 1768) & 0 & 1 & 0 & 1 & 3 & 1.0 & fr & $\mathrm{mz}$ & zo & $\mathrm{cm}$ \\
\hline 68 & Abax carinatus (Duftschmid, 1812) & 0 & 0 & 0 & 3 & 4 & 1.4 & fr & $\mathrm{mz}$ & zo & $\mathrm{cm}$ \\
\hline 69 & A. parallelus (Duftschmid, 1812) & 0 & 0 & 0 & 5 & 3 & 1.6 & fr & $\mathrm{mz}$ & zo & $\mathrm{cm}$ \\
\hline 70 & A. parallelepipedus (Piller et Mitterpacher, 1783) & 0 & 0 & 0 & 4 & 3 & 1.4 & fr & $\mathrm{mz}$ & zo & $\mathrm{cm}$ \\
\hline 71 & Molops piceus (Panzer, 1793 ) & 0 & 0 & 0 & 2 & 2 & 0.8 & fr & $\mathrm{mz}$ & $\mathrm{zf}$ & $\mathrm{cm}$ \\
\hline 72 & Poecilus crenuliger Chaudoir, 1876 & 2 & 1 & 1 & 0 & 0 & 0.8 & st & $\mathrm{mx}$ & zo & $\mathrm{cm}$ \\
\hline 73 & P. cupreus (Linnaeus, 1912) & 4 & 2 & 2 & 3 & 1 & 2.4 & $\mathrm{pt}$ & $\mathrm{mz}$ & zo & df \\
\hline 74 & P. koyi Germar, 1823 (=sericeus F.-W., 1824) & 2 & 1 & 1 & 0 & 0 & 0.8 & md & $\mathrm{mz}$ & zo & $\mathrm{cm}$ \\
\hline 75 & P. puncticollis (Dejean, 1828) & 1 & 0 & 0 & 0 & 0 & 0.2 & st & $\mathrm{mx}$ & zo & $\mathrm{cm}$ \\
\hline 76 & P. lepidus (Leske, 1787) & 0 & 0 & 0 & 3 & 0 & 0.6 & fr & $\mathrm{mz}$ & zo & $\mathrm{cm}$ \\
\hline 77 & P. punctulatus (Schaller, 1783) & 2 & 1 & 1 & 1 & 0 & 1.0 & md & $\mathrm{mz}$ & zo & df \\
\hline 78 & P. versicolor (Sturm, 1824) & 4 & 4 & 4 & 3 & 1 & 3.2 & md & $\mathrm{mz}$ & zo & $\mathrm{cm}$ \\
\hline 79 & Pterostichus anthracinus (Illiger, 1798) & 2 & 1 & 1 & 1 & 1 & 1.2 & md & $\mathrm{mh}$ & zo & $\operatorname{lm}$ \\
\hline 80 & P. diligens (Sturm, 1824) & 0 & 0 & 0 & 1 & 0 & 0.2 & $\mathrm{fr}$ & $\mathrm{mh}$ & zo & $\operatorname{lm}$ \\
\hline 81 & P. elongatus (Duftschmid, 1812) & 1 & 0 & 0 & 0 & 0 & 0.2 & md & $\mathrm{mh}$ & zo & sl \\
\hline 82 & P. gracilis (Dejean, 1828) & 1 & 0 & 1 & 1 & 0 & 0.6 & md & $\mathrm{mh}$ & zo & $\operatorname{lm}$ \\
\hline 83 & P. macer Marsham, 1802 & 1 & 0 & 0 & 0 & 0 & 0.2 & md & $\mathrm{mz}$ & zo & $\mathrm{cm}$ \\
\hline 84 & P. melanarius (Illiger, 1798) & 5 & 5 & 5 & 5 & 5 & 5.0 & $\mathrm{pt}$ & $\mathrm{mz}$ & zo & df \\
\hline 85 & P. melas (Creutzer, 1799) & 1 & 1 & 0 & 2 & 0 & 0.8 & md & $\mathrm{mz}$ & zo & $\operatorname{lm}$ \\
\hline 86 & P. minor (Gyllenhal, 1827) & 1 & 0 & 0 & 1 & 4 & 1.2 & md & $\mathrm{mh}$ & zo & $\operatorname{lm}$ \\
\hline 87 & P. niger (Schaller, 1783) & 3 & 2 & 1 & 3 & 5 & 2.8 & $\mathrm{fr}$ & $\mathrm{mz}$ & zo & $\operatorname{lm}$ \\
\hline 88 & P. nigrita (Paykull, 1790) & 2 & 1 & 0 & 1 & 4 & 1.6 & md & $\mathrm{mh}$ & zo & $\operatorname{lm}$ \\
\hline 89 & P. oblongopunctatus (Fabricius, 1787) & 5 & 5 & 4 & 5 & 5 & 4.8 & fr & $\mathrm{mz}$ & zo & $\operatorname{lm}$ \\
\hline 90 & P. ovoideus (Sturm, 1824) & 3 & 4 & 3 & 0 & 0 & 2.0 & md & $\mathrm{mz}$ & zo & $\mathrm{cm}$ \\
\hline 91 & P. strenuus (Panzer, 1797) & 2 & 4 & 1 & 3 & 4 & 2.8 & $\mathrm{md}$ & $\mathrm{mz}$ & zo & $\mathrm{cm}$ \\
\hline 92 & P. quadrifoveolatus (Letzner, 1852) & 0 & 0 & 0 & 0 & 3 & 0.6 & fr & $\mathrm{mz}$ & zo & $\operatorname{lm}$ \\
\hline 93 & P. rhaeticus Heer, 1837 & 0 & 0 & 0 & 0 & 2 & 0.4 & fr & $\mathrm{mz}$ & zo & $\operatorname{lm}$ \\
\hline 94 & P. vernalis (Panzer, 1796) & 2 & 0 & 1 & 1 & 0 & 0.8 & md & $\mathrm{mh}$ & zo & $\mathrm{cm}$ \\
\hline 95 & Stomis pumicatus (Panzer, 1796) & 1 & 1 & 0 & 1 & 1 & 0.8 & $\mathrm{fr}$ & $\mathrm{mz}$ & zo & $\mathrm{cm}$ \\
\hline 96 & Calathus ambiguus (Paykull, 1790) & 3 & 2 & 4 & 3 & 0 & 2.4 & $\mathrm{pt}$ & $\mathrm{mz}$ & zo & df \\
\hline 97 & C. distinguendus Chaudoir, 1846 & 0 & 3 & 0 & 0 & 0 & 0.6 & st & $\mathrm{mx}$ & zo & $\mathrm{cm}$ \\
\hline 98 & C. erratus (C.R. Sahlberg, 1827) & 2 & 1 & 2 & 1 & 0 & 1.2 & md & $\mathrm{mz}$ & zo & $\operatorname{lm}$ \\
\hline 99 & C. fuscipes (Goeze, 1777) & 4 & 1 & 4 & 4 & 0 & 2.6 & $\mathrm{pt}$ & $\mathrm{mz}$ & zo & $\mathrm{df}$ \\
\hline 100 & C. melanocephalus (Linnaeus, 1758) & 2 & 3 & 2 & 3 & 0 & 2.0 & $\mathrm{pt}$ & $\mathrm{mz}$ & zo & df \\
\hline 101 & Dolichus halensis (Schaller, 1783) & 1 & 1 & 1 & 2 & 0 & 1.0 & md & $\mathrm{mz}$ & zo & $\mathrm{cm}$ \\
\hline 102 & Laemostenus terricola (Herbst, 1783)* & 1 & 2 & 1 & 0 & 0 & 0.8 & md & $\mathrm{mz}$ & zo & $\mathrm{cm}$ \\
\hline 103 & Taphoxenus gigas (Fischer von Waldheim, 1823) & 1 & 0 & 0 & 0 & 0 & 0.2 & st & $\mathrm{mx}$ & zo & $\mathrm{cm}$ \\
\hline 104 & Agonum duftschmidi J. Schmidt, 1994 & 0 & 1 & 1 & 1 & 1 & 0.8 & md & $\mathrm{mh}$ & zo & $\operatorname{lm}$ \\
\hline 105 & A. gracilipes (Duftschmid, 1812) & 0 & 1 & 1 & 0 & 0 & 0.4 & md & $\mathrm{mh}$ & zo & df \\
\hline
\end{tabular}




\begin{tabular}{|c|c|c|c|c|c|c|c|c|c|c|c|}
\hline No & Species & Dnipro & Donetsk & Kharkiv & Kyiv & Lviv & $\begin{array}{l}\text { Total for } \\
\text { five cities }\end{array}$ & $\begin{array}{c}\text { Biotopic } \\
\text { preferred } \\
\text { range }\end{array}$ & $\begin{array}{l}\text { Humidi- } \\
\text { fication } \\
\text { preferred } \\
\text { range } \\
\end{array}$ & $\begin{array}{c}\text { Trophic } \\
\text { characte- } \\
\text { rization }\end{array}$ & $\begin{array}{c}\text { Soil } \\
\text { texture } \\
\text { preferred } \\
\text { range } \\
\end{array}$ \\
\hline 106 & A. lugens (Duftschmid, 1812) & 2 & 0 & 0 & 1 & 0 & 0.6 & $\mathrm{md}$ & $\mathrm{mh}$ & zo & $\operatorname{lm}$ \\
\hline 107 & A. versutum (Sturm, 1824) & 1 & 0 & 1 & 0 & 0 & 0.4 & md & $\mathrm{mh}$ & zo & $\operatorname{lm}$ \\
\hline 108 & A. viduum (Panzer, 1797) & 1 & 0 & 0 & 1 & 0 & 0.4 & md & $\mathrm{mh}$ & zo & $\operatorname{lm}$ \\
\hline 109 & Oxypselaphus obscurum (Herbst, 1784) & 2 & 0 & 0 & 0 & 5 & 1.4 & fr & $\mathrm{mh}$ & zo & $\operatorname{lm}$ \\
\hline 110 & Anchomenus dorsalis (Pontoppidan, 1763) & 3 & 5 & 4 & 4 & 1 & 3.4 & $\mathrm{pt}$ & $\mathrm{mz}$ & zo & df \\
\hline 111 & Limodromus assimilis (Paykull, 1790) & 0 & 2 & 1 & 4 & 5 & 2.4 & fr & $\mathrm{mz}$ & zo & $\operatorname{lm}$ \\
\hline 112 & L. krynickii Sperk, 1835 & 2 & 0 & 0 & 0 & 0 & 0.4 & fr & $\mathrm{mh}$ & zo & $\operatorname{lm}$ \\
\hline 113 & Synuchus vivalis (Illiger, 1798) & 1 & 1 & 1 & 1 & 1 & 1.0 & fr & $\mathrm{mz}$ & zo & $\operatorname{lm}$ \\
\hline 114 & Amara aenea (De Geer, 1774) & 5 & 2 & 2 & 5 & 1 & 3.0 & $\mathrm{pt}$ & $\mathrm{mz}$ & $\mathrm{fz}$ & df \\
\hline 115 & A. apricaria (Paykull, 1790) & 2 & 1 & 3 & 2 & 0 & 1.6 & $\mathrm{pt}$ & $\mathrm{mz}$ & $\mathrm{fz}$ & df \\
\hline 116 & A. aulica (Panzer,1797) & 1 & 2 & 1 & 1 & 0 & 1.0 & md & $\mathrm{mz}$ & $\mathrm{zf}$ & $\mathrm{cm}$ \\
\hline 117 & A. bifrons (Gyllenhal, 1810) & 0 & 0 & 3 & 1 & 0 & 0.8 & $\mathrm{md}$ & $\mathrm{mz}$ & $\mathrm{fz}$ & $\mathrm{sl}$ \\
\hline 118 & A. brunnea (Gyllenhal, 1810) & 0 & 0 & 0 & 1 & 0 & 0.2 & fr & $\mathrm{mz}$ & $\mathrm{fz}$ & $\operatorname{lm}$ \\
\hline 119 & A. communis (Panzer, 1797) & 3 & 1 & 1 & 3 & 0 & 1.6 & fr & $\mathrm{mz}$ & $\mathrm{fz}$ & $\operatorname{lm}$ \\
\hline 120 & A. consularis (Duftschmid, 1812) & 1 & 1 & 1 & 2 & 0 & 1.0 & $\mathrm{pt}$ & $\mathrm{mz}$ & $\mathrm{zf}$ & df \\
\hline 121 & A. convexior Stephens, 1828 & 0 & 4 & 2 & 2 & 0 & 1.6 & md & $\mathrm{mz}$ & $\mathrm{zf}$ & $\operatorname{lm}$ \\
\hline 122 & A. convexiuscula (Marsham, 1802) & 1 & 0 & 0 & 1 & 0 & 0.2 & md & $\mathrm{mz}$ & $\mathrm{zf}$ & $\mathrm{cm}$ \\
\hline 123 & A. crenata (Dejean, 1828 ) & 1 & 0 & 0 & 0 & 0 & 0.2 & st & $\mathrm{mx}$ & $\mathrm{fz}$ & $\mathrm{cm}$ \\
\hline 124 & A. equestris (Duftschmid, 1812) & 1 & 1 & 1 & 0 & 0 & 0.6 & md & $\mathrm{mz}$ & $\mathrm{zf}$ & $\mathrm{cm}$ \\
\hline 125 & A. eurynota (Panzer, 1797) & 2 & 2 & 0 & 1 & 0 & 1.0 & md & $\mathrm{mz}$ & $\mathrm{fz}$ & df \\
\hline 126 & A. famelica C. Zimmermann, 1832 & 1 & 0 & 0 & 1 & 0 & 0.4 & $\mathrm{md}$ & $\mathrm{mz}$ & $\mathrm{fz}$ & $\operatorname{lm}$ \\
\hline 127 & A. familiaris (Duftschmid, 1812) & 2 & 1 & 2 & 3 & 0 & 1.6 & $\mathrm{md}$ & $\mathrm{mz}$ & $\mathrm{fz}$ & df \\
\hline 128 & A. fulva (O. Müller, 1776) & 1 & 0 & 1 & 0 & 0 & 0.4 & md & $\mathrm{mz}$ & $\mathrm{fz}$ & sd \\
\hline 129 & A. ingenua (Duftschmid, 1812) & 2 & 1 & 0 & 1 & 0 & 0.8 & md & $\mathrm{mz}$ & fz & $\mathrm{sl}$ \\
\hline 130 & A. littorea C.G. Thomson, 1857 & 0 & 0 & 1 & 1 & 0 & 0.4 & $\mathrm{md}$ & $\mathrm{mz}$ & $\mathrm{fz}$ & $\operatorname{lm}$ \\
\hline 131 & A. lunicollis Schiǿdte, 1837 & 1 & 0 & 0 & 1 & 0 & 0.4 & $\mathrm{md}$ & $\mathrm{mz}$ & $\mathrm{fz}$ & $\operatorname{lm}$ \\
\hline 132 & A. majuscula (Chaudoir, 1850) & 1 & 0 & 0 & 1 & 0 & 0.4 & $\mathrm{md}$ & $\mathrm{mz}$ & $\mathrm{fz}$ & sl \\
\hline 133 & A. municipalis (Duftschmid, 1812) & 1 & 1 & 0 & 0 & 0 & 0.4 & md & $\mathrm{mz}$ & $\mathrm{fz}$ & $\operatorname{lm}$ \\
\hline 134 & A. ovata (Fabricius, 1792) & 2 & 2 & 2 & 2 & 0 & 1.6 & $\mathrm{md}$ & $\mathrm{mz}$ & $\mathrm{fz}$ & df \\
\hline 135 & A. plebeja (Gyllenhal, 1810) & 0 & 0 & 0 & 2 & 0 & 0.4 & fr & $\mathrm{mz}$ & $\mathrm{fz}$ & $\operatorname{lm}$ \\
\hline 136 & A. similata (Gyllenhal, 1810) & 3 & 3 & 3 & 3 & 0 & 2.4 & $\mathrm{pt}$ & $\mathrm{mz}$ & $\mathrm{fz}$ & df \\
\hline 137 & A. spreta Dejean, 1831 & 0 & 0 & 0 & 1 & 0 & 0.2 & md & $\mathrm{mz}$ & $\mathrm{fz}$ & $\mathrm{cm}$ \\
\hline 138 & A. tibialis (Paykull, 1798) & 0 & 0 & 0 & 1 & 0 & 0.2 & $\mathrm{md}$ & $\mathrm{mz}$ & $\mathrm{fz}$ & $\operatorname{lm}$ \\
\hline 139 & A. tricuspidata Dejean, 1831 & 1 & 0 & 0 & 1 & 0 & 0.4 & $\mathrm{md}$ & $\mathrm{mz}$ & $\mathrm{fz}$ & $\mathrm{cm}$ \\
\hline 140 & Zabrus spinipes steveni (Fischer von Waldheim, 1817) & 2 & 1 & 0 & 0 & 0 & 0.6 & st & $\mathrm{mx}$ & $\mathrm{fz}$ & $\mathrm{cm}$ \\
\hline 141 & Z. tenebrioides (Goeze, 1777) & 2 & 1 & 1 & 0 & 0 & 0.8 & st & $\mathrm{mz}$ & $\mathrm{ff}$ & $\mathrm{cm}$ \\
\hline 142 & Anisodactylus binotatus (Fabricius, 1787) & 2 & 1 & 2 & 1 & 1 & 1.4 & $\mathrm{md}$ & $\mathrm{mz}$ & $\mathrm{zf}$ & $\operatorname{lm}$ \\
\hline 143 & A. nemorivagus (Duftschmid, 1812) & 1 & 0 & 0 & 1 & 0 & 0.4 & fr & $\mathrm{mz}$ & $\mathrm{zf}$ & $\operatorname{lm}$ \\
\hline 144 & A. poeciloides pseudoaeneus Dejean, 1829 & 1 & 0 & 0 & 0 & 0 & 0.2 & st & $\mathrm{mz}$ & $\mathrm{zf}$ & sl \\
\hline 145 & A. signatus (Panzer, 1797) & 2 & 1 & 1 & 1 & 1 & 1.2 & $\mathrm{pt}$ & $\mathrm{mz}$ & $\mathrm{zf}$ & df \\
\hline 146 & Acupalpus meridianus (Linnaeus, 1767) & 1 & 1 & 1 & 2 & 0 & 1.0 & $\mathrm{pt}$ & $\mathrm{mz}$ & $\mathrm{fz}$ & df \\
\hline 147 & A. exiguus (Dejean, 1829) & 0 & 0 & 0 & 1 & 1 & 0.4 & fr & $\mathrm{mh}$ & $\mathrm{fz}$ & $\operatorname{lm}$ \\
\hline 148 & Diachromus germanus (Linnaeus, 1758) & 1 & 0 & 0 & 0 & 0 & 0.2 & md & $\mathrm{mz}$ & $\mathrm{zf}$ & $\mathrm{cm}$ \\
\hline 149 & Bradycellus verbasci (Duftschmid, 1812) & 0 & 0 & 0 & 1 & 0 & 0.2 & fr & $\mathrm{mz}$ & $\mathrm{fz}$ & $\operatorname{lm}$ \\
\hline 150 & Dicheirotrichus ustulatus (Dejean, 1829) & 1 & 0 & 0 & 0 & 0 & 0.2 & md & $\mathrm{mh}$ & $\mathrm{zf}$ & $\mathrm{sl}$ \\
\hline 151 & Stenolophus abdominalis persicus (Mannerheim, 1844) & 2 & 0 & 0 & 0 & 0 & 0.4 & lt & $\mathrm{mh}$ & $\mathrm{zf}$ & sl \\
\hline 152 & S. discophorus (Fischer von Waldheim, 1823) & 1 & 0 & 0 & 0 & 0 & 0.2 & lt & $\mathrm{mh}$ & $\mathrm{fz}$ & $\mathrm{sl}$ \\
\hline 153 & S. mixtus (Herbst, 1784) & 2 & 1 & 1 & 1 & 1 & 1.2 & lt & $\mathrm{mh}$ & $\mathrm{zf}$ & $\mathrm{sl}$ \\
\hline 154 & S. proximus (Dejean, 1829) & 1 & 0 & 0 & 0 & 0 & 0.2 & lt & $\mathrm{mh}$ & $\mathrm{zf}$ & $\mathrm{sl}$ \\
\hline 155 & S. teutonus (Schrank, 1781) & 2 & 0 & 1 & 1 & 0 & 0.8 & lt & $\mathrm{mh}$ & $\mathrm{zf}$ & $\mathrm{sl}$ \\
\hline 156 & Harpalus affinis (Schrank, 1781) & 3 & 2 & 5 & 2 & 0 & 2.4 & md & $\mathrm{mz}$ & $\mathrm{zf}$ & df \\
\hline 157 & H. amplicollis Menetries, 1848 & 2 & 0 & 2 & 1 & 0 & 0.8 & st & $\mathrm{mx}$ & $\mathrm{zf}$ & $\mathrm{cm}$ \\
\hline 158 & H. anxius Duftschmid, 1812 & 2 & 2 & 2 & 3 & 0 & 1.8 & $\mathrm{pt}$ & $\mathrm{mz}$ & $\mathrm{zf}$ & df \\
\hline 159 & H. atratus (Latreille, 1804) & 0 & 1 & 2 & 0 & 0 & 0.6 & fr & $\mathrm{mz}$ & $\mathrm{zf}$ & $\operatorname{lm}$ \\
\hline 160 & H. autumnalis (Duftschmid, 1812) & 0 & 0 & 1 & 0 & 0 & 0.2 & md & $\mathrm{mz}$ & $\mathrm{fz}$ & $\mathrm{cm}$ \\
\hline 161 & H. calceatus (Duftschmid, 1812) & 2 & 1 & 0 & 1 & 0 & 0.8 & $\mathrm{pt}$ & $\mathrm{mz}$ & $\mathrm{zf}$ & $\mathrm{cm}$ \\
\hline 162 & H. caspius (Steven, 1806) & 1 & 1 & 1 & 1 & 0 & 0.8 & md & $\mathrm{mz}$ & $\mathrm{zf}$ & $\operatorname{lm}$ \\
\hline 163 & H. distinguendus (Duftschmid, 1812) & 5 & 2 & 5 & 4 & 1 & 3.4 & $\mathrm{pt}$ & $\mathrm{mz}$ & $\mathrm{zf}$ & df \\
\hline 164 & H. flavicornis Dejean, 1829 & 0 & 1 & 0 & 0 & 0 & 0.2 & md & $\mathrm{mz}$ & $\mathrm{zf}$ & $\operatorname{lm}$ \\
\hline 165 & H. froelichi Sturm, 1818 & 1 & 1 & 1 & 1 & 0 & 0.8 & $\mathrm{pt}$ & $\mathrm{mz}$ & $\mathrm{zf}$ & $\mathrm{cm}$ \\
\hline 166 & H. fuscipalpis (Sturm, 1818) & 2 & 0 & 0 & 0 & 0 & 0.4 & st & $\mathrm{mz}$ & $\mathrm{zf}$ & $\mathrm{cm}$ \\
\hline 167 & H. griseus (Panzer, 1797) & 3 & 1 & 5 & 2 & 0 & 2.2 & $\mathrm{pt}$ & $\mathrm{mz}$ & $\mathrm{zf}$ & $\mathrm{cm}$ \\
\hline 168 & H. hirtipes(Panzer, 1796) & 0 & 0 & 1 & 0 & 0 & 0.2 & md & $\mathrm{mz}$ & $\mathrm{zf}$ & $\mathrm{cm}$ \\
\hline 169 & $\begin{array}{l}\text { H. laeviceps Zetterstedt, } 1828 \\
\text { (=quadripunctatus Dej., 1829) }\end{array}$ & 0 & 2 & 1 & 2 & 0 & 1.0 & fr & $\mathrm{mz}$ & $\mathrm{zf}$ & $\operatorname{lm}$ \\
\hline 170 & H. latus (Linnaeus, 1758) & 3 & 4 & 3 & 2 & 0 & 2.4 & fr & $\mathrm{mz}$ & $\mathrm{zf}$ & $\operatorname{lm}$ \\
\hline 171 & H. luteicornis (Duftschmid, 1812) & 0 & 0 & 1 & 1 & 1 & 0.6 & fr & $\mathrm{mz}$ & $\mathrm{zf}$ & $\operatorname{lm}$ \\
\hline 172 & H. melancholicus Dejean, 1829 & 1 & 0 & 0 & 0 & 0 & 0.2 & st & $\mathrm{mx}$ & $\mathrm{zf}$ & $\operatorname{lm}$ \\
\hline 173 & H. modestus Dejean, 1829 & 0 & 0 & 2 & 0 & 0 & 0.4 & st & $\mathrm{mx}$ & $\mathrm{fz}$ & $\mathrm{cm}$ \\
\hline 174 & H. picipennis Duftschmid, 1812 & 1 & 1 & 1 & 0 & 0 & 0.6 & $\mathrm{md}$ & $\mathrm{mx}$ & $\mathrm{zf}$ & df \\
\hline 175 & H. politus Dejean, 1829 & 0 & 0 & 1 & 0 & 0 & 0.2 & $\mathrm{md}$ & $\mathrm{mx}$ & $\mathrm{zf}$ & $\mathrm{cm}$ \\
\hline 176 & H. progrediens (Schauberger, 1922) & 0 & 0 & 0 & 0 & 1 & 0.2 & fr & $\mathrm{mz}$ & $\mathrm{zf}$ & $\mathrm{cm}$ \\
\hline 177 & H. pumilus (Sturm, 1818) & 0 & 1 & 2 & 2 & 0 & 1.0 & $\mathrm{md}$ & $\mathrm{mz}$ & $\mathrm{fz}$ & $\mathrm{cm}$ \\
\hline 178 & H. pygmaeus Dejean, 1829 & 0 & 0 & 1 & 0 & 0 & 0.2 & $\mathrm{fr}$ & $\mathrm{mz}$ & $\mathrm{zf}$ & $\mathrm{cm}$ \\
\hline
\end{tabular}




\begin{tabular}{|c|c|c|c|c|c|c|c|c|c|c|c|}
\hline No & Species & Dnipro & Donetsk & Kharkiv & Kyiv & Lviv & $\begin{array}{l}\text { Total for } \\
\text { five cities }\end{array}$ & $\begin{array}{l}\text { Biotopic } \\
\text { preferred } \\
\text { range }\end{array}$ & $\begin{array}{l}\text { Humidi- } \\
\text { fication } \\
\text { preferred } \\
\text { range } \\
\end{array}$ & $\begin{array}{l}\text { Trophic } \\
\text { characte- } \\
\text { rization }\end{array}$ & $\begin{array}{c}\text { Soil } \\
\text { texture } \\
\text { preferred } \\
\text { range } \\
\end{array}$ \\
\hline 179 & H. rubripes (Duftschmid, 1812) & 0 & 1 & 1 & 3 & 0 & 1.0 & $\mathrm{md}$ & $\mathrm{mz}$ & $\mathrm{zf}$ & df \\
\hline 180 & H. rufipes (De Geer, 1774) & 5 & 5 & 5 & 4 & 1 & 4.0 & $\mathrm{pt}$ & $\mathrm{mz}$ & $\mathrm{zf}$ & $\mathrm{df}$ \\
\hline 181 & H. serripes (Quensel, 1806) & 1 & 1 & 1 & 1 & 0 & 0.8 & md & $\mathrm{mz}$ & $\mathrm{zf}$ & df \\
\hline 182 & H. servus (Duftschmid, 1812) & 1 & 0 & 0 & 0 & 0 & 0.2 & st & $\mathrm{mx}$ & $\mathrm{fz}$ & sl \\
\hline 183 & H. signaticornis (Duftschmid, 1812) & 0 & 0 & 1 & 0 & 0 & 0.2 & md & $\mathrm{mz}$ & $\mathrm{zf}$ & $\operatorname{lm}$ \\
\hline 184 & H. smaragdinus (Duftschmid, 1812) & 3 & 1 & 3 & 1 & 0 & 1.6 & md & $\mathrm{mz}$ & $\mathrm{zf}$ & df \\
\hline 185 & H. subcylindricus Dejean, 1829 & 0 & 1 & 1 & 0 & 0 & 0.4 & st & $\mathrm{mx}$ & $\mathrm{zf}$ & $\mathrm{cm}$ \\
\hline 186 & H. tardus (Panzer, 1797) & 2 & 3 & 4 & 3 & 0 & 2.4 & md & $\mathrm{mz}$ & $\mathrm{fz}$ & $\mathrm{cm}$ \\
\hline 187 & H. tenebrosus Dejean, 1829 & 0 & 0 & 1 & 0 & 0 & 0.2 & st & $\mathrm{mz}$ & $\mathrm{zf}$ & $\mathrm{cm}$ \\
\hline 188 & H. xanthopus winkleri Schauberger, 1923 & 2 & 1 & 3 & 1 & 0 & 1.4 & md & $\mathrm{mz}$ & $\mathrm{zf}$ & $\operatorname{lm}$ \\
\hline 189 & H. zabroides Dejean, 1829 & 1 & 1 & 0 & 0 & 0 & 0.4 & md & $\mathrm{mx}$ & $\mathrm{zf}$ & $\mathrm{cm}$ \\
\hline 190 & Ophonus azureus (Fabricius, 1775) & 1 & 1 & 1 & 2 & 0 & 1.0 & md & $\mathrm{mz}$ & $\mathrm{zf}$ & df \\
\hline 191 & O. cordatus (Duftschmid, 1812) & 0 & 0 & 1 & 0 & 0 & 0.2 & md & $\mathrm{mz}$ & $\mathrm{zf}$ & $\operatorname{lm}$ \\
\hline 192 & O. diffinis (Dejean, 1829) & 0 & 0 & 1 & 0 & 0 & 0.2 & md & $\mathrm{mx}$ & $\mathrm{zf}$ & $\operatorname{lm}$ \\
\hline 193 & O. laticollis (Mannerheim, 1825) & 1 & 2 & 0 & 1 & 0 & 0.8 & md & $\mathrm{mz}$ & $\mathrm{zf}$ & df \\
\hline 194 & O. puncticollis (Paykull, 1798) & 0 & 1 & 0 & 2 & 0 & 0.6 & md & $\mathrm{mz}$ & $\mathrm{zf}$ & $\operatorname{lm}$ \\
\hline 195 & O. rufibarbis (Fabricius, 1792) & 0 & 1 & 0 & 0 & 1 & 0.4 & md & $\mathrm{mz}$ & $\mathrm{zf}$ & $\operatorname{lm}$ \\
\hline 196 & O. subquadratus (Dejean, 1829) & 0 & 1 & 0 & 0 & 0 & 0.2 & st & $\mathrm{mz}$ & $\mathrm{zf}$ & $\operatorname{lm}$ \\
\hline 197 & O. shaubergerianus Puel, 1937 & 0 & 0 & 0 & 1 & 0 & 0.2 & md & $\mathrm{mx}$ & $\mathrm{zf}$ & $\operatorname{lm}$ \\
\hline 198 & O. rufibarbis (Fabricius, 1792) & 1 & 0 & 1 & 0 & 1 & 0.6 & md & $\mathrm{mz}$ & $\mathrm{zf}$ & $\operatorname{lm}$ \\
\hline 199 & Trichotichnus laevicollis (Duftschmid, 1812) & 0 & 0 & 0 & 0 & 3 & 0.6 & fr & $\mathrm{mz}$ & $\mathrm{zf}$ & $\operatorname{lm}$ \\
\hline 200 & Panagaeus bipustulatus (Fabricius, 1775) & 1 & 2 & 2 & 1 & 0 & 1.2 & md & $\mathrm{mz}$ & zo & $\operatorname{lm}$ \\
\hline 201 & P. cruxmajor (Linnaeus, 1758) & 1 & 0 & 0 & 0 & 0 & 0.2 & fr & $\mathrm{mz}$ & zo & $\operatorname{lm}$ \\
\hline 202 & Chlaenius aeneocephalus (Dejean, 1826) & 1 & 1 & 0 & 0 & 0 & 0.4 & st & $\mathrm{mz}$ & zo & $\mathrm{cl}$ \\
\hline 203 & Ch. nitidulus (Schrank, 1781) & 0 & 0 & 0 & 1 & 1 & 0.4 & lt & $\mathrm{mh}$ & zo & $\operatorname{lm}$ \\
\hline 204 & Ch. tristis (Schaller, 1783) & 1 & 1 & 1 & 0 & 0 & 0.6 & md & $\mathrm{mz}$ & zo & df \\
\hline 205 & Ch. vestitus (Paykull, 1790) & 0 & 1 & 0 & 1 & 0 & 0.4 & lt & $\mathrm{hm}$ & zo & sl \\
\hline 206 & Dinodes decipiens (Dufour, 1820) & 1 & 1 & 0 & 0 & 0 & 0.4 & st & $\mathrm{mx}$ & zo & $\mathrm{cm}$ \\
\hline 207 & Oodes gracilis (A. Villa et G.B. Villa, 1833) & 1 & 0 & 0 & 0 & 0 & 0.2 & md & $\mathrm{mh}$ & $\mathrm{zf}$ & $\operatorname{lm}$ \\
\hline 208 & O. helopioides (Fabricius, 1792) & 1 & 0 & 1 & 0 & 1 & 0.6 & $\mathrm{md}$ & $\mathrm{mh}$ & $\mathrm{zf}$ & $\operatorname{lm}$ \\
\hline 209 & Badister bullatus (Schrank, 1798) & 2 & 3 & 2 & 2 & 0 & 1.8 & md & $\mathrm{mz}$ & zo & $\operatorname{lm}$ \\
\hline 210 & B. dilatatus (Chaudoir, 1837) & 0 & 1 & 1 & 0 & 0 & 0.4 & md & $\mathrm{hm}$ & zo & $\operatorname{lm}$ \\
\hline 211 & B. lacertosus Sturm, 1815 & 1 & 0 & 0 & 0 & 1 & 0.4 & fr & $\mathrm{mh}$ & zo & $\operatorname{lm}$ \\
\hline 212 & B. meridionalis Puel, 1925 & 1 & 0 & 0 & 0 & 0 & 0.2 & $\mathrm{md}$ & $\mathrm{mh}$ & zo & $\operatorname{lm}$ \\
\hline 213 & B. sodalis (Duftschmid, 1812) & 0 & 0 & 0 & 1 & 1 & 0.4 & fr & $\mathrm{hm}$ & zo & $\operatorname{lm}$ \\
\hline 214 & B. unipustulatus Bonelli, 1813 & 1 & 1 & 1 & 1 & 0 & 0.8 & md & $\mathrm{mh}$ & zo & $\operatorname{lm}$ \\
\hline 215 & Licinus cassideus (Fabricius, 1792) & 1 & 0 & 1 & 0 & 0 & 0.4 & md & $\mathrm{mz}$ & zo & $\mathrm{cm}$ \\
\hline 216 & L. depressus (Paykull, 1790) & 2 & 2 & 2 & 1 & 0 & 1.4 & md & $\mathrm{mz}$ & zo & df \\
\hline 217 & L. silphoides (P. Rossi, 1790) & 0 & 1 & 0 & 0 & 0 & 0.2 & st & $\mathrm{mx}$ & zo & $\operatorname{lm}$ \\
\hline 218 & Masoreus wetterhalli (Gyllenhal, 1813)* & 1 & 0 & 2 & 0 & 0 & 0.6 & fr & $\mathrm{mz}$ & $\mathrm{zf}$ & $\operatorname{lm}$ \\
\hline 219 & Odacantha melanura (Linnaeus, 1767) & 1 & 0 & 0 & 0 & 0 & 0.2 & $\mathrm{md}$ & $\mathrm{mz}$ & zo & $\operatorname{lm}$ \\
\hline 220 & Lebia cyanocephala (Linnaeus, 1758) & 0 & 1 & 0 & 1 & 0 & 0.4 & md & $\mathrm{mz}$ & zo & df \\
\hline 221 & Demetrias monostigma Samouelle, 1819 & 1 & 0 & 0 & 0 & 0 & 0.2 & md & $\mathrm{mz}$ & zo & $\operatorname{lm}$ \\
\hline 222 & Dromius quadrimaculatus (Linnaeus, 1758) & 1 & 0 & 1 & 1 & 0 & 0.6 & fr & $\mathrm{mz}$ & zo & $\operatorname{lm}$ \\
\hline 223 & Microlestes maurus (Sturm, 1827) & 0 & 1 & 1 & 0 & 0 & 0.4 & md & $\mathrm{mz}$ & $\mathrm{zf}$ & $\operatorname{lm}$ \\
\hline 224 & M. minutulus (Goeze, 1777) & 3 & 3 & 2 & 2 & 0 & 2.0 & $\mathrm{pt}$ & $\mathrm{mz}$ & zo & df \\
\hline 225 & M. negrita (Wollaston, 1854) & 0 & 1 & 1 & 0 & 0 & 0.4 & st & $\mathrm{mx}$ & zo & $\mathrm{cm}$ \\
\hline 226 & M. plagiatus (Duftschmid, 1812) & 1 & 0 & 0 & 0 & 0 & 0.2 & md & $\mathrm{mx}$ & zo & $\mathrm{cm}$ \\
\hline 227 & Syntomus foveatus (Fourcroy, 1785) & 1 & 0 & 0 & 0 & 0 & 0.2 & md & $\mathrm{mz}$ & zo & $\mathrm{cm}$ \\
\hline 228 & S. obscuroguttatus (Duftschmid, 1812) & 1 & 1 & 0 & 0 & 0 & 0.4 & md & $\mathrm{mz}$ & zo & $\mathrm{cm}$ \\
\hline 229 & S. pallipes (Dejean, 1825) & 0 & 0 & 1 & 1 & 0 & 0.4 & md & $\mathrm{mz}$ & zo & $\operatorname{lm}$ \\
\hline 230 & S. truncatellus (Linnaeus, 1761) & 2 & 1 & 1 & 1 & 0 & 1.0 & md & $\mathrm{mz}$ & zo & $\mathrm{cm}$ \\
\hline 231 & Cymindis cingulata Dejean, 1825 & 0 & 0 & 0 & 0 & 1 & 0.2 & fr & $\mathrm{mz}$ & zo & $\operatorname{lm}$ \\
\hline 232 & Drypta dentata (Rossi, 1790) & 1 & 0 & 1 & 1 & 0 & 0.6 & md & $\mathrm{mz}$ & zo & $\operatorname{lm}$ \\
\hline 233 & Polystichus connexus (Geoffroy, 1785) & 0 & 1 & 0 & 0 & 0 & 0.2 & st & $\mathrm{mx}$ & zo & sd \\
\hline 234 & Brachinus brevicollis Motschulsky, 1844 & 1 & 0 & 0 & 0 & 0 & 0.2 & st & $\mathrm{mz}$ & zo & sl \\
\hline 235 & B. crepitans (Linnaeus, 1758) & 2 & 2 & 1 & 1 & 0 & 1.2 & md & $\mathrm{mz}$ & zo & df \\
\hline 236 & B. ejaculans Fischer von Waldheim, 1829 & 1 & 0 & 0 & 0 & 0 & 0.2 & md & $\mathrm{mh}$ & zo & $\operatorname{lm}$ \\
\hline \multirow[t]{7}{*}{237} & B. elegans Chaudoir, 1842 & 0 & 1 & 0 & 0 & 0 & 0.2 & md & $\mathrm{m}$ & zo & $\operatorname{lm}$ \\
\hline & $\begin{array}{l}\text { Total number of species per city (number of spe- } \\
\text { cies/their share in the number of all species) } \\
\text { including: }\end{array}$ & $\begin{array}{c}156 / \\
100.0\end{array}$ & $\begin{array}{c}119 / \\
100.0\end{array}$ & $\begin{array}{c}125 / \\
100.0\end{array}$ & $\begin{array}{c}137 / \\
100.0\end{array}$ & $\begin{array}{c}66 / \\
100.0\end{array}$ & $\begin{array}{c}237 / \\
100.0\end{array}$ & - & - & - & - \\
\hline & Eudominants & $5 / 3.2$ & $4 / 3.4$ & $6 / 4.8$ & $4 / 2.9$ & $8 / 12.1$ & $3 / 1.2$ & - & - & - & - \\
\hline & Dominants & $4 / 2.6$ & $6 / 5.0$ & $6 / 4.8$ & $12 / 8.8$ & $5 / 7.6$ & $5 / 2.1$ & - & - & - & - \\
\hline & Subdominants & $13 / 8.3$ & $9 / 7.6$ & $15 / 12.0$ & $17 / 12.4$ & $9 / 13.6$ & $25 / 10.5$ & - & - & - & - \\
\hline & Recedents & $47 / 30.2$ & $24 / 20.2$ & $24 / 19.2$ & $31 / 22.6$ & $5 / 7.6$ & $53 / 22.4$ & - & - & - & - \\
\hline & Occasional species (subrecedents) & $87 / 55.7$ & $76 / 63.8$ & $74 / 59.2$ & $73 / 53.3$ & $39 / 59.1$ & $151 / 63.8$ & - & - & - & - \\
\hline
\end{tabular}

Note: abundance for separate cities (explanation of the levels of abundance - see the section Material and Methods): 0 - species is absent from the collections, 1 - occasional species, 2 - rare species (recedent), 3 - subdominant species, 4 - common species (dominant), 5 - abundant species (eudominant); in general for all the metropolises (points): 4.0-5.0 - eudominants, 3.0-3.9- dominants, 1.7-2.9 - subdominants, 1.0-1.6-recedents, $0.2-0.8$ - subrecedents; * - species is not reported for Ukraine in the catalogue of Palearctic (Catalogue of Palearctic Coleoptera, 2017); biotopic distribution: pt - polytopic, fr - forest, st - steppe, md - meadow, lt - littoral; hygropreferendum: mx - mesoxerophylous, $\mathrm{mz}$ - mesophylous, hm - hygromezophilous, $\mathrm{mh}$ - mesohygrophylous; trophic specialization: $\mathrm{ff}$ - phytophagous, zf - zoophytophagous, $\mathrm{fz}$ - phytozoophagous, zo zoophagous; preferred mechanical composition of soils: $\mathrm{df}$ - different soil texture, sd - sandy, sl - sand loamy, $\operatorname{lm}$ - loam, $\mathrm{cm}$ - clay loamy, cl - clay. 
Table 2

Number of species of ground beetles of different ecological groups for metropolises (species in total, in brackets - the number of common species)

\begin{tabular}{|c|c|c|c|c|c|c|c|}
\hline $\begin{array}{c}\text { Type of } \\
\text { classification }\end{array}$ & Ecological groups & Dnipro & Donetsk & Kharkiv & Kyiv & Lviv & Total \\
\hline \multirow{5}{*}{$\begin{array}{l}\text { Habitat } \\
\text { preference }\end{array}$} & pt-polytopic & $24(11)$ & $23(6)$ & $24(11)$ & $25(13)$ & $10(1)$ & $25(16)$ \\
\hline & $\mathrm{fr}-$ forest & $21(4)$ & $17(6)$ & $20(5)$ & $39(14)$ & $35(17)$ & 59 (7) \\
\hline & st-steppe & $15(0)$ & $13(1)$ & $7(1)$ & $1(0)$ & $0(0)$ & $23(10)$ \\
\hline & md-meadow & $87(7)$ & $64(6)$ & $71(11)$ & $67(7)$ & $19(4)$ & $119(0)$ \\
\hline & lt-littoral & $9(0)$ & $2(0)$ & $3(0)$ & $5(0)$ & $2(0)$ & $11(0)$ \\
\hline \multirow{4}{*}{ Hydropreference } & $\mathrm{mx}$ - mesoxerophylous & $13(0)$ & $12(1)$ & $8(0)$ & $2(0)$ & $0(0)$ & $23(0)$ \\
\hline & mz-mesophylous & $111(22)$ & $95(16)$ & $97(27)$ & $111(33)$ & $48(17)$ & $165(31)$ \\
\hline & $\mathrm{hm}$-hygromesophylous & $1(0)$ & $2(0)$ & $1(0)$ & $3(0)$ & $2(0)$ & $6(0)$ \\
\hline & $\mathrm{mh}-$ mesohygrophylous & $31(0)$ & $10(1)$ & $19(0)$ & $21(1)$ & $16(5)$ & $43(2)$ \\
\hline \multirow{4}{*}{$\begin{array}{l}\text { Trophic } \\
\text { specialisation }\end{array}$} & zo-zoophagous; & $97(13)$ & $72(13)$ & $72(15)$ & $82(25)$ & $52(21)$ & $146(24)$ \\
\hline & $\mathrm{fz}$-phytozoophagous & $20(3)$ & $13(2)$ & $13(4)$ & $23(5)$ & $2(0)$ & $30(2)$ \\
\hline & zf-zoophytophagous & $38(6)$ & $33(3)$ & $39(10)$ & $31(4)$ & $12(1)$ & $60(7)$ \\
\hline & ff-phytophagous & $1(0)$ & $1(0)$ & $1(0)$ & $0(0)$ & $0(0)$ & $1(0)$ \\
\hline \multirow{6}{*}{$\begin{array}{l}\text { Soil texture } \\
\text { preference }\end{array}$} & df-different soil texture & $38(13)$ & $39(7)$ & $38(14)$ & $37(16)$ & $11(1)$ & $41(17)$ \\
\hline & sd - sand & $4(0)$ & $1(0)$ & $2(0)$ & $3(0)$ & $0(0)$ & $6(0)$ \\
\hline & sl-sandy loamy & $13(0)$ & $3(0)$ & $4(1)$ & $8(1)$ & $2(0)$ & $16(0)$ \\
\hline & $\mathrm{lm}$-loam & $44(4)$ & $30(4)$ & $36(4)$ & $49(7)$ & $30(11)$ & $85(6)$ \\
\hline & $\mathrm{cm}$-clay loamy & $53(5)$ & $43(7)$ & $45(7)$ & $40(11)$ & $23(10)$ & $85(10)$ \\
\hline & $\mathrm{cl}$ - clay & $4(0)$ & $3(0)$ & $0(0)$ & $0(0)$ & $0(0)$ & $4(0)$ \\
\hline
\end{tabular}

Among the studied communities of beetles, four types of ecological classification were distinguished: according to biotopic confinement, hygropreferendum, trophic characteristic and soil structure preference (Table 1,2, Fig. 1). This division to some extent is conditional, but in general characterizes ecological peculiarities of carabidofauna of one or the other metropolises. Their analysis is presented in the discussion of the results (as well as the analysis of the taxonomic structure).

\section{Discussion}

Caraboid beetles (Coleoptera, Caraboidea) recorded in urbocenoses in all the metropolises are one of the dominating groups of coleopterans. In the epigeal habitat, they exceeded the quantitative parameters of such large familities such as Staphylinidae and Curculionidae (Nazarenko \& Petrenko, 2008; Brygadyrenko, 2015b; Komaromi et al., 2018; Nazarenko et al., 2020; Putchkov et al., 2020). The number of species of caraboid beetles was almost twice the number of species of these families, and according to abundance they were also dominant (up to $60 \%$ of all beetles in the litter fauna of urbocenoses).

On the basis of abundance of species of Carabidae in general for all metropolises, only three species were identified to typical eudominants: Harpalus rufipes, Pterostichus melanarius and P. oblongopunctatus. Common (dominants) were five species: Amara aenea, Anchomenus dorsalis, Calathus fuscipes, Harpalus distinguendus and Poecilus versicolor. Subdominants were represented by 25 species (10.5\% of species composition of ground beetles): Amara similata, Asaphidion flavipes, Badister bullatus, Bembidion lampros, B. properans, Broscus cephalotes, Calathus ambiguus, C. melanocephalus, Carabus cancellatus, C. coriaceus, C. granulatus, Cylindera germanica, Harpalus affinis, H. anxius, H. griseus, H. latus, H. tardus, Limodromus assimilis, Microlestes minutulus, Nebria brevicollis, Notiophilus palustris, Poecilus cupreus, Pterostichus niger, $P$. ovoideus and $P$. strenuus. That is, in general, 33 species could be identified (13.9\% of total number of species of Carabidae) to common species of ground beetles (eudominant, dominant and subdominant species) recorded in urbocenoses of all the metropolises we surveyed. Rare species (recedents) were represented by 53 species $(22.4 \%)$ and occasional (subrecedents) - 151 (63.7\%) species (Tables 1,2).

The situation for the cities varied. The number of eudominants was 4 (Donetsk, Kyiv) to 6-8 species (Kharkiv, Lviv). The highest (but practically equal) parameters were seen for dominant species and subdominants (Tables 1,2). However, the number of rare and subrecedent species for separate cities varied more greatly. Their higher number was observed in Dnipro (47 and 87 species or respectively $30 \%$ and $55 \%$ of all the species of ground beetles). In Donetsk, Kharkiv and Kyiv, these groups included 24-31 rare and 73-76 occasional species (respectively 19$23 \%$ and $53-64 \%$ of all the species of ground beetles). In the carabidofauna of Lviv, the number and the share of rare and occasional species was minimum (respectively 5 rare and 39 occasional species). Moreover, in separate cities, sometimes the numerically dominant species were those which had a comparatively low average share in total for all metropolies taken as a whole. Apart from the abovementioned mass and common species same for the metropolises, one of the eudominants in the parks of Kharkiv was Carabus nemoralis, in Donetsk - Amara convexior, Notiophilus palustris and Pterostichus strenuus; Dnipro Poecilus cupreus and Bembidion lampros, Kyiv - species of Abax genus and some of Carabus (C. cancellatus, C. convexus, C. excellens). Only in Lviv, the dominants were Oxypselaphus obscurum, Carabus arcensis and C. glabratus, absent in other metropolises. Moreover, in the latter two cities, the common species were also the ones not recorded in other metropolises (Nebria brevicollis, Limodromus assimilis). The species Pterostichus minor and $P$. nigrita also dominated only in Lviv, similarly to Amara communis in Dnipro and Kyiv.

The indicators of number we presented both for separate species and overall, indicate significant faunistic oligodominance of the carabidofaunas in different metropolises (especially at the level of abundant species), the specificity of their carabidofauna. In general, the total level of species diversity of Carabidae in urbocenoses was closer to that in agrocenoses of different regions of Ukraine according to the main parameters of biodiversity than to taxonomically more evenly natural ecosystems, especially in the comparative aspect of their faunas (Putchkov, 2018).

There were some faunistically interesting discoveries of some species of ground beetles. As many times mentioned earlier (Putchkov, 2018), seven species - Clivina collaris, C. fossor, Elaphrus uliginosus, Laemostenus terricola, Masoreus wetterhalli, Notiophilus germinyi and Scarites terricola were not reported for Ukraine in the latest Catalogue of Palearctic Coleoptera (2017). This does not mean that they were not indicated for the country earlier (Komaromi et al., 2018; Putchkov, 2018), but indicates insufficient familiarization of authors of the Catalogue with separate literature sources on carabidofauna of Ukraine. Many data on these findings were published in articles and reports in Ukrainian or Russian languages, complicating the research on them for foreign coleopterologists (mainly European).

However, regarding peculiarities of geographic distribution in Ukraine of a number of species of ground beetles (around 20), certain elaborations were made during the comparison with the latest check-list of Caraboidea of Ukraine (Putchkov, 2018). Therefore, discoveries of ten species (Amara famelica, A. majuscula, Anisodactylus nemorivagus, Badister lacertosus, Blemus discus, Limodromus krynickii, Pterostichus minor), recorded in urbocenoses of Dnipro, and also Asaphidion pallipes, Tachyta nana (Dnipro and Donetsk) and Harpalus laeviceps (Donetsk) were new for the steppe zone of Ukraine. Species Chlaenius aeneocephalus and Brachinus brevicollis were recorded for the first time in the Right Bank part of the northern subzone of Steppe Ukraine (Dnipro). Northward range extensions were confirmed for the typical 
steppe species Taphoxenus gigas (south of Foresst Steppe), and Stenolophus abdominalis, indicated for the far south of Ukraine, was reported for the first time for the northern steppe subzone (both species in Dnipro). The species Microlestes negrita was found for the first time in the Forest Steppe (Kharkiv), and Masoreus wetterhalli (Kharkiv) and Syntomus foveatus (Dnipro), known earlier for the forest zone and the south of the Steppe, were for the first time recorded in the northern subzone

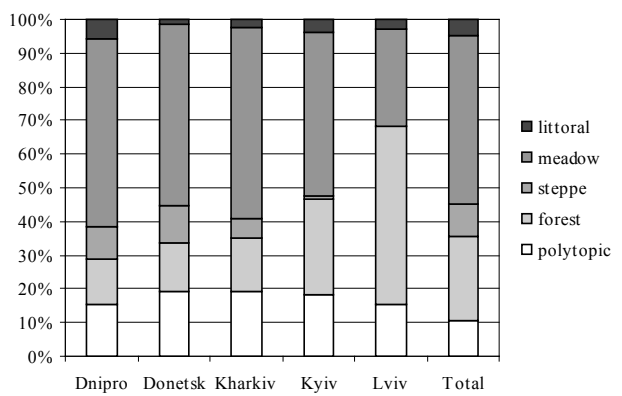

$a$

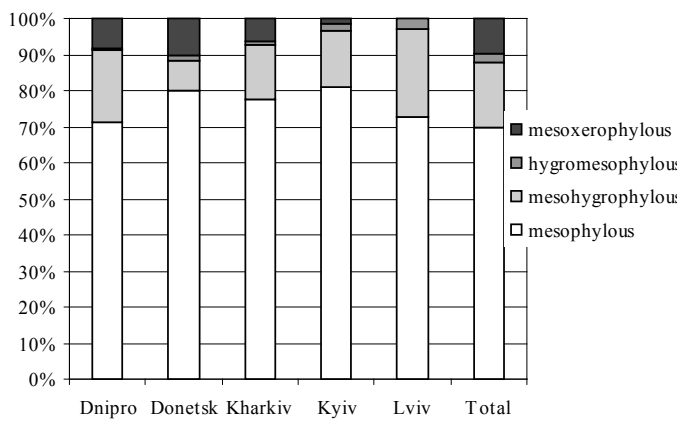

$c$

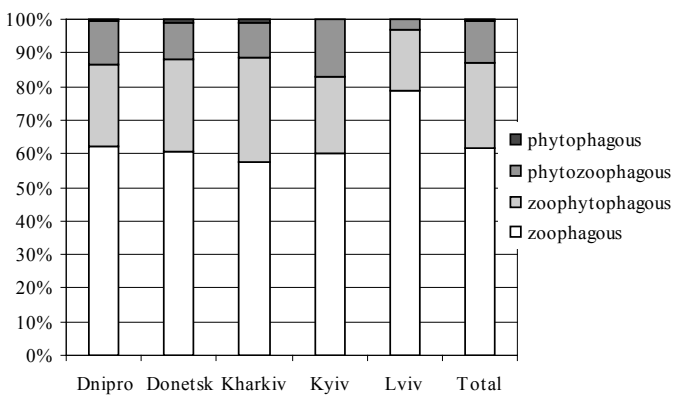

$e$

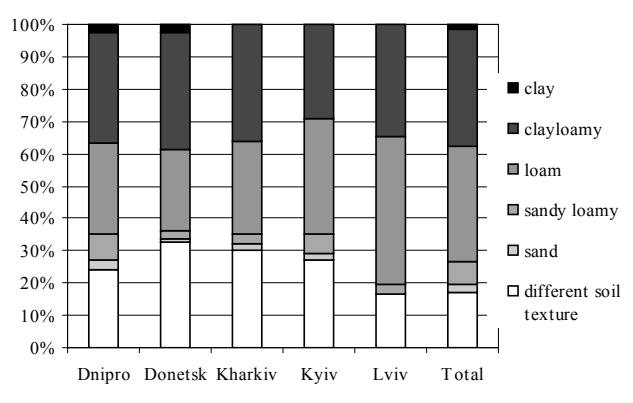

$g$ and the Forest Steppe. Furthermore, discoveries of Calathus distinguendus (Donetsk), distribution of which in Ukraine is studied insuficiently, were confirmed in the northern steppe subzone (Putchkov \& Aleksandrowicz, 2020). All the given information about new findings was quite predictable (taking into consideration the general ranges of these species), but indicates the insufficient degree of the research on the distribution of many species of ground beetles in Ukraine.

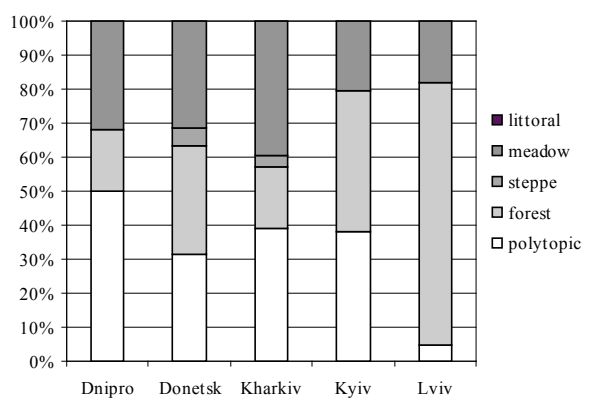

$b$

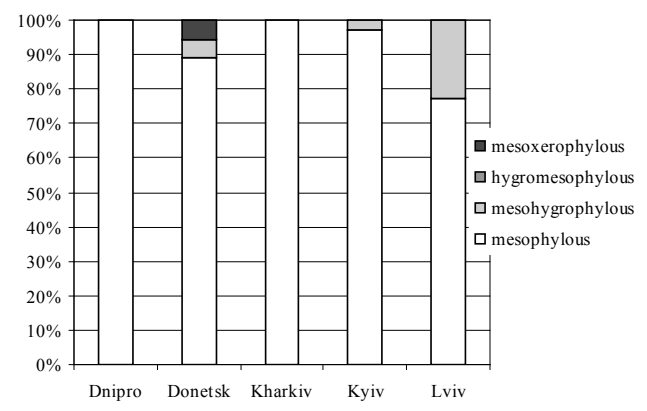

$d$

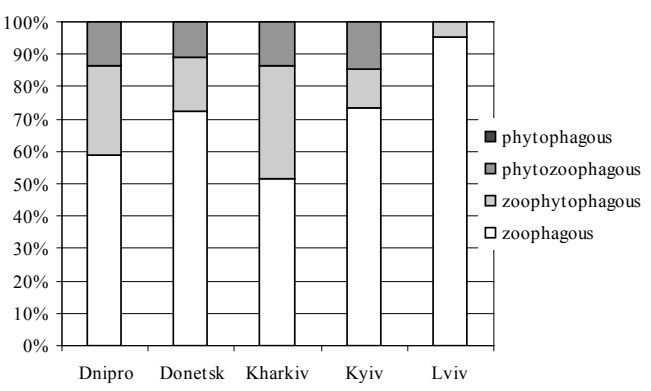

$f$

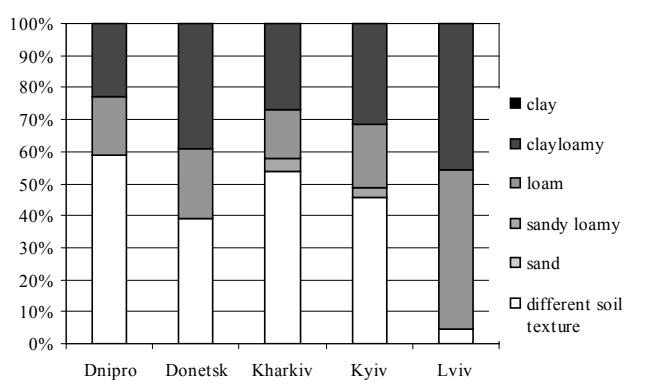

$h$

Fig. 1. Ratio (\%) of the number of species of ground beetles and tiger beetles of different ecological groups in metropolises $(a, c, e, g)$ and abundant species $(b, d, f, h)$ in the cities of Ukraine

Differences in qualitative and quantitative parameters of the communities of Carabidoidea in the cities revealed significant differences in their faunistic similarity (Fig. 2a). In the comparison of all carabidofauna, the Jaccard coefficients ranged within $0.20-0.60$. The lowest similarity was seen between Lviv and the other cities. More similar were the carabidofaunas of Kharkiv and Donetsk (around 0.60), these parameters were slightly lower between Dnipro and Kyiv (around 0.50). A similar situation was revealed also by comparing the abundant species of ground beetles (eudominant, dominant and subdominant species) in different metropolises, but with far higher indicators (Fig. 2b). Jaccard coefficient equaled 0.32-0.87. Similarity of Caraboidea of Kyiv and most cities was 0.72, and between Dnipro, Donetsk and Kharkiv reached over 0.83 . The highest similarity was seen between Donetsk and Kharkiv (0.87), which is not surprising (considering the proximity of these 
metropolises, especially according to longitude). Minimum indicators were seen for Lviv and other metropolises ( 0.32$)$, but they were one and a half times higher than in the comparison of all the carabidofauna (Fig. $3 a$ ). Average value of the variation of the Jaccard coefficient accounted for $0.40-0.55$, which could suggest sufficient similarity of Caraboidea of all the studied metropolises.

The levels of the indicators given above could indicate comparatively low differences in the main component of the carabidofauna of most cities as a result of comparatively close zonal conditions. Kyiv and Kharkiv are located in the forest steppe zone, and Dnipro and Donetskin the northern steppe subzone neighbouring with the Forest Steppe. Faunistic originality of carabidofauna of Lviv is due to its location in the subzone of broad-leaves forests, where the taxonomic composition of ground beetles is quite specific compared with other geographic regions of Ukraine. A certain role also belongs to the Carpathians, some specific representatives of whose fauna penetrate the western part of the forest zone of Ukraine.

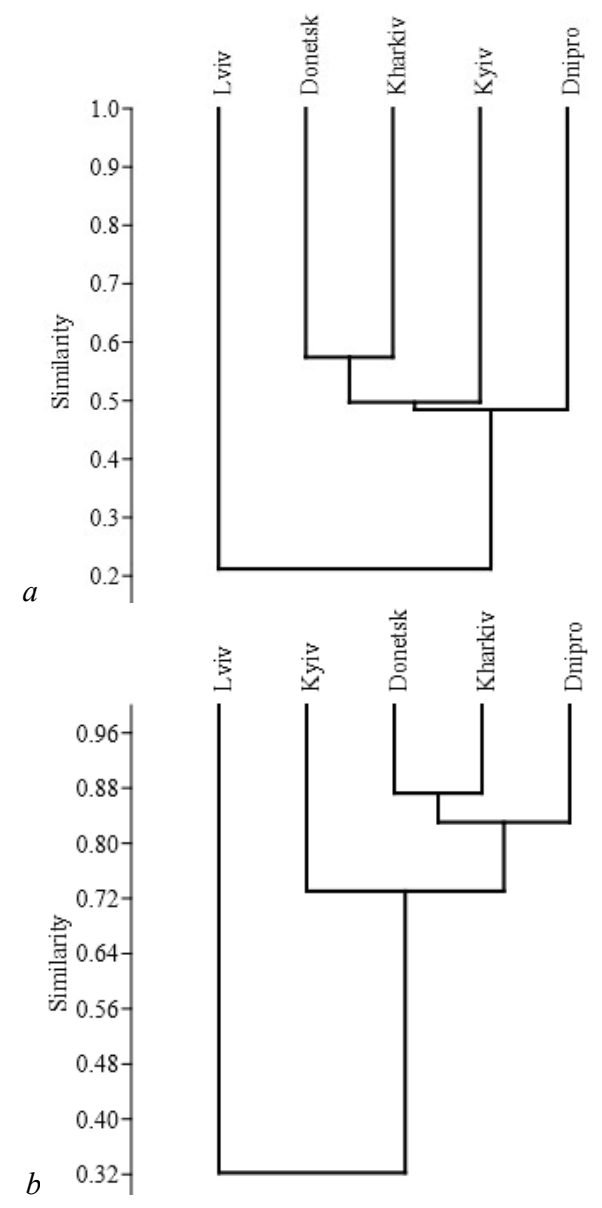

Fig. 2. Cluster analysis of similarity according to the Jacquard index of the entire carabidofauna of the megalopolises $(a)$ and the abundant species $(b)$

A no less important aspect which underlies the differences in abundance and faunistic similarity is also the peculiarities of the ecological structure of the community of ground beetles in metropolises (Tables 1, 2). According to their lifestyle, peculiarities of diet and habitat distribution of species, all of the Caraboidea recorded in the urbocenoses could be divided according to several main ecological principals: biotopic distribution, hygropreferendum, trophic specialization and soil characteristics of their environments. To some extent, such approaches are subjective, and in some studies of the other researchers, the same species could be identified to different (but close) groups or subgroups (Brygadyrenko, 2015a, 2015c, 2016).

The structure of carabidofauna was quite diverse regarding biotopic preferendum, and therefore was considered only within four large groups: polytopic, meadow, forest and littoral. Based on the overall compo- sition of the communities of ground beetles of the cities we studied, the faunistically most numerous were meadow (119) and forest elements (59 species, Fig. 1). Among the polytopic group, 25 species were noted, the steppe group was represented by 23 species, and among littoral (coastal), 11 species were recorded (Table 2, Fig. 1). However, in quantitative aspect, almost in all the metropolises we surveyed, the polytopic group dominated, in which common species formed almost the half (except Lviv). Among the forest complex in Dnipro, Donetsk and Kharkiv, 4-6 common species were recorded (13-16\% of all carabidofauna), whereas in Kyiv and Lviv their number equaled almost third (14 17 species, 28-53\%, Fig. 1). Despite the fact that meadow elements dominated in almost all cities (64-87 species), abundant elements of this group accounted for 8-15\% (4-11 species). Most of the steppe species (13-15) were seen in Dnipro and Donetsk, fewer in Kharkiv (7) and only a couple in Kyiv (Fig. 1). Abundant species among this group were not found, though in southern cities, certain steppe elements could reach the level of subdominants (for example Calathus distinguendus in Donetsk or Zabrus tenebrioides in Dnipro). The number of littoral elements ranged from two (Donetsk, Lviv) to nine (Dnipro), but no abundant species were recorded among them (Table 2, Fig. 1).

According to the general (point) assessement of abundance of abundant species of ground beetles, in total in the studied cities, the basis for carabidofauna comprised polytopic (16 species) and meadow elements (10 species), less - forest inhabitants, represented by 7 species (Table 2; Fig. 1). At the same time, among eudominant and dominants, polytopic elements were represented by six species, whereas meadow and forest - one species each.

The data above in many aspects vary. The complexity of the analysis of biotopic structure among such large groups as forest and meadow species (less - among the litorral group) is due to the presence of some transitional groups (around 10), which (for facilitating the analysis) are intentionally not distinguished and discussed. Most species of these subgroups were recorded as recedents or subrecedents, but certain representatives sometimes were recorded as subdominants in separate cities. Therefore, among the forest group, forest-shrub groups (about 20 species) were recorded, many of which were sporadically subdominants (Amara plebeja, Anisodactylus binotatus, Asaphidion pallipes, Calosoma inquisitor, Carabus violaceus, Harpalus xanthopus winkleri, Leistus ferrugineus, Masoreus wetterhalli, Odacantha melanura, Stomis pumicatus). Among the flood plain-forest subgroup in urbocenoses, around 10 rare species were recorded (Badister lacertosus, B. sodalis, Oxypselaphus obscurum and others). There were also recorded forest-marsh elements (Acupalpus exiguus, Carabus menetriesi, C. variolosus). To the meadow group, almost 40 representatives of the subgroup of meadow-steppe species were identified, some of which were subdominants and also dominants in some cities (Carabus excellens, C. scabriusculus, Harpalus caspius, H. pumilus, H. serripes, Licinus depressus, Poecilus koyi, P. punctulatus, most species of the Metophonus subgenus), and also around 20 meadow-shrub species (Amara communis, A. familiaris, A. ingenua, A. ovata, Brachinus crepitans, Calathus erratus, Harpalus rubripes, H. smaragdinus, H. tardus, Ophonus laticollis many Synto$m u s$ ). In the group of littoral species, not only typical elements were included (for example most of species of Bembidion and Stenolophus genera), but also inhabitants of humid flood-plain meadows - in total about 10 rare species (Brachinus ejaculans, Chlaenius tristis, most of the species of Agonum, Badister and Oodes genera). However, the diversity of littoral species in some cities (for example, Dnipro and Kyiv) is obviously higher due to the quite rich littoral carabidofauna, study of which was not included in the objectives of these researches.

According to other ecological characteristics, a handful of halophilic species of ground beetles were also recorded - Anisodactylus poeciloides pseudoaeneus, Brachinus brevicollis, Dicheirotrichus ustulatus, Poecilus puncticollis, Tachys scutellaris, mainly in separate urbocenoses of Dnipro.

Regarding moisture, the eudominant species were typical mesophilous (165 species, i.e. over third of all the ground beetles). At the same time, there was an especially high number of mesophilous abundant species (16-33 species for different cities, around 94\% of all carabidofauna (Table 2, Fig. 1). In general, the ratio of mesophilous for 
different cities varied insignificantly. Mesoxerophilous and mesohygrophilous were represented by 23 and 43 species, almost all of which were recorded as rare or occasional (Fig. 1). Only three mesohygrophilous Nebria brevicollis, Oxypselaphus obscurum and Pterostichus minor (some of dominants in Kyiv and Lviv) were sporadically recorded as subdominants (Table 1). The share of mesohygrophilous in Dnipro and Lviv was slightly higher than in Kharkiv and Kyiv, but nonetheless minimum in Donetsk. However, for mesoxerophilous, the reverse situation was seen: they were observed more often in Dnipro and Donetsk, more rarely - in Kyiv and Kharkiv and were absent in Lviv.

According to trophic specialization, four groups were distinguished, of which the dominant were zoophages of different specialization (146 species in total and over 60\% of all the carabidofauna). Among abundant species, zoophagous were also eudominants ( 24 of 33 species, Table 2, Fig. 1). They all were represented both by obligatory predators (species of the tribes Bembidiini, Brachinini, Broscini, Carabini, Chlaenini, Cicindelini, Licinini, Lebiini, Nebriini and Notiophilini) and principal zoophages of the tribes Pterostichini, Platinini, Sphodrini and some others (Korolev \& Brygadyrenko, 2014; Putchkov, 2018). The zoophages were also fairly diverse (60 species, over $25 \%$ of all the carabidofauna), including seven species identified to abundant (Fig. 1). The major share of this group comprised species of the Harpalini tribe. Among the phytozoohages, 30 species were seen (mostly of Zabrini tribe), including two species (Amara aenea and $A$. similata) identified to abundant (Table 2, Fig. 1).One species, Zabrus tenebrioides, was identified to phytophages; it was rarely recorded as subdominant in the urbocenoses of Dnipro. However, the closely related $Z$. spinipes was classified to phytozoophages and seen in the cities as rare or occasional. In general, proportion of the three main trophic groups in the cities differed insignificantly (Table 2, Fig. 1).

Most complicated and to a large degree conditional was the division of ground beetles into groups regarding the confinement to different types of soils and their mechanical composition. More accurate data could be obtained only as a result of studying the distribution and abundance of larvae of ground beetles, data on which are so far insufficient (we used the data on 82 species). In the absence of such material, the species were divided into soil groups provisionally, taking into consideration the peculiarities of quantitative distribution and abundance of imagoes both according to our observations and based on a number of literature sources generalized in some studies (Sharova, 1981; Brygadyrenko, 2015a, 2015c, 2016; Putchkov, 2018). In total, six groups of species of Carabidae were distinguished in terms of preference for one or the other mechanical composition of soil (Fig. 1). The most diverse groups comprised species which prefer loamy and clayey-loamy soils 85 species in each, in total accounting for over $70 \%$ of species composition. However, abundant elements among these groups were only 6 of 10 species respectively (Table 2, Fig. 1). Quite richly represented was the group of ground beetles without clear preferences for mechanical composition of soil, to which 41 species were identified (17.3\% of all the carabidofauna). However, by the number of common species, this group dominated and included 17 species - i.e. over the half of the abundant and common ground beetles. Six and sixteen species, among which no species were found abundant for the cities, were identified to the inhabitants of sandy and sabulous soils. The number of species which are associated with heavy clayey soils was minimum, most likely due to poor degree of study on such inhabitants (Table 2, Fig. 1).

In general, as well as according to taxonomic composition (Fig. 1), the ecological structure of the communities of ground beetles of most Ukrainian metropolises (based on the main characteristics) was more specific in the comparison of all carabidofauna (i.e. both abundant and rare species). However, comparison of only abundant species in the cities revealed quite close similarity of their carabidofaunas, especially by proportions of different ecological groups (differences for different metropolises equaled more than $10 \%$ - Table 2, Fig. 1). The only exception was Lviv, where the carabidofauna was the most original. This could be explained by specificity of local carabidocomplexes and the quite fragmented study on the fauna of ground beetles conducted in separate years in a minimum number of urbocenoses. More detailed studies on Carabidae in the cities of Western Ukraine, similarly to Southern regions (for example Odessa), would provide better understanding of the formation of the population of ground beetles of urban landscapes.

The above-mentioned peculiarities of taxonomic and ecological structures cause a number of differences in qualitative and quantitative structures of the Caraboidea community, originality of their fauna for some cities. In general, the range of ecological groups was minimum in Lviv, but maximum in Dnipro and Kyiv (Table 1, 2). Typical representative of caraboid beetles in urbocenoses of the main metropolises of Ukraine could be considered the polytopic and meadow mesophile zoophage (to a less extent forest), but also ecologically flexible species well adapted to living in urban plantations.

\section{Conclusion}

In total, in the urbocenoses, 237 species of Caraboidea were recorded (Table 1), belonging to 63 genera and two families - Carabidae (231 species, 61 genera) and Cicindelidae (6 species, 2 genera), which accounts for almost one third of taxonomic compound of Caraboidea superfamily in Ukraine. According to the abundance of Carabidae in all the metropolises we studied, 33 species were identified to abundant (about $25 \%$ of their total number). Eudominants were represented by three species: Harpalus rufipes, Pterostichus melanarius and P. oblongopunctatus. Five species were common: Amara aenea, Anchomenus dorsalis, Calathus fuscipes, Harpalus distinguendus and Poecilus versicolor. Subdominants were represented by 25 species: Amara similata, Asaphidion flavipes, Badister bullatus, Bembidion lampros, B. properans, Broscus cephalotes, Calathus ambiguus, C. melanocephalus, Carabus cancellatus, C. coriaceus, C. granulatus, Cylindera germanica, Harpalus affinis, H. anxius, H. griseus, H. latus, H. tardus, Limodromus assimilis, Microlestes minutulus, Nebria brevicollis, Notiophilus palustris, Poecilus cupreus, Pterostichus niger, $P$. ovoideus and P. strenuus. A total of 53 species (22.4\%) were identified to rare (recendents), and $151(63.7 \%)$ species to occasional (subrecendents). For separate cities, the number of eudominants was 4 (Donetsk, Kyiv) to 6-8 (Kharkiv, Lviv). In Dnipro, 47 and 48 rare and occasional species respectively were recorded, while in Donetsk, Kharkiv and Kyiv these groups included 24-31 rare and 73-76 occasional species.

Finds of ten species - Amara famelica, A. majuscula, Anisodactylus nemorivagus, Badister lacertosus, Blemus discus, Limodromus krynickii, Pterostichus minor (recorded in urbocenoses of Dnipro), and also Asaphidion pallipes, Tachyta nana (Dnipro and Donetsk) and Harpalus laeviceps (Donetsk) were new for the steppe zone of Ukraine. Chlaenius aeneocephalus and Brachinus brevicollis were for the first time recorded in the Right Bank part of the northern subzone of Steppe Ukraine, and Stenolophus abdominalis reported for the far south of Ukraine was for the first time reported for the Northern steppe subzone (Dnipro). Microlestes negrita was new for the Forest Steppe (Kharkiv). The species Masoreus wetterhalli (Kharkiv) and Syntomus foveatus (Dnipro) known earlier for the forest zone and south of the steppe zone were for the first time reported for the Northern subzone of the Steppe and Forest Steppe.

The levels of faunistic similarity of Caraboidea for different metropolises ranged within $0.20-0.60$. Most similar (by coefficient of Jaccard) were the carabidofaunas of Kharkiv and Donetsk, slightly less in Dnipro and Kyiv (around 0.50). Minimum indicators were observed for Lviv and other cities (about 0.20). A similar situation was also revealed by the comparison of abundant species of ground beetles in different metroplises, but with much higher indicators (0.32-0.87).

According to species composition, the most numerous were meadow (119) and forest (59 species). A total of 25 species was identified to the polytopic group, 23 to steppe, and 11 to littoral. Almost in all metropolises, the polytopic group dominated, among which the abundant species formed almost half (except Lviv). Among the forest complex in Dnipro, Donetsk and Kharkiv, 4-6 abundant species were recorded, whereas in Kyiv and Lviv, their quantity equaled more than one third (14-17 species). Among the meadow group, 4-11 species were abundant (more of them in Dnipro and Donetsk). Abundant species among the steppe and littoral groups were absent. Regarding moisture, eudominants were mesophiles (165 species), among which 31 common species 
were recorded. According to trophic specialization, zoophagous species dominated (146 species and over $60 \%$ of all carabidofauna, including 24 abundant species. Among zoophytophages, 60 species were recorded (more than $25 \%$ of all the carabidofauna), seven of which were abundant. Phytozoopages were represented by 30 species (two common). In relation to the mechanical composition of soil, the highest diversity was seen among the species which prefer loamy and clayloamy soils -85 species in each group. However, abundant elements among these groups were 6 and 10 species respectively. The group of ground beetles without clear preference to mechanical composition of soil was represented by 41 species, but by the number of abundant elements (17 species), it was the dominant group. Four, six and sixteen species were identified as inhabitants of heavy clayey, sandy and sabolous soils respectively, with no abundant elements. At the level of abundant species, in most metropolises, the ecological structure was more similar than shown by the analysis of all species of ground beetles.

The conducted studies allow us to analyze qualitative and quantitative characteristics of communities of Caraboidea in metropolises of Ukraine, evaluate their faunistic similarity, and in brief characterize the ecological structure. On the basis of the analyzed data, the typical representatives of caraboid beetles in metropolises of Ukraine could be considered the polytopic or meadow (less - forest) mesophile zoophages or zoophytophages which prefer loamy-clayey soils or without preference to one or the other mechanical composition of soil.

The authors express sincere gratitude to the Candidate of Biological Sciences V.V. Martynov (Botanical Garden, Donetsk) for providing material on Caraboidea in urbocenoses of Donetsk for analysis, and also Candidate of Biological Sciences A. G. Shatrovsky (Karasin Kharkiv National University, Kharkiv) for collecting ground beetles in some plantations of Kharkiv. This work was supported by the Ministry of Education and Science of Ukraine (grant 0118U003303).

\section{References}

Aleksandrowicz, O. R. (1997). Sklad i naselnitstva zhuzhalei (Coleoptera, Carabidae) Minska [Composition and population of ground beetles (Coleoptera, Carabidae) of Minsk]. Minsk Maxim Tank Belarusian State Pedagogical University, Minsk, 3, 75-80 (in Belarussian).

Aleksandrowicz, O. R. (2014). Zhuzhelitsy (Coleoptera, Carabidae) zapada lesnoy zony Russkoy Ravniny (fauna, zoogeografiya, ekologiya, faunogenez) [Ground beetles (Coleoptera, Carabidae) of the West of the Forest zone of the Russian Plain (fauna, zoogeography, ecology, faunogenesis)]. Lambert Academic Publishing, Saarbrücken (in Russian).

Avtaeva, T A., Sukhodolskaya, R. A., Eremeeva, N. I., Gordienko, T. A., Vodunon, N. P., Lechieva, M. I., Kushaliev, S. A., \& Kuropyatnik, K. N. (2019). Ground beetles (Coleoptera, Carabidae) assemblages features formation in large and medium industrial cities of Russia (the case study in Kazan, Kemerovo and Grozny). In: International scientific and practical conference "AgroSMART Smart solutions for agriculture”. KnE Life Sciences. Pp. 232-245.

Balkenhol, B., Flisse, J., \& Zucchi, H. (1991). Investigations into carabid beetles and spiders (Carabidae and Araneida) in an urban quarry - to the problem of habitat isolation. Pedobiologia, 35(3), 153-162.

Brygadyrenko, V. V. (2015a). Evaluation of the ecological niche of some abundant species of the subfamily Platyninae (Coleoptera, Carabidae) against the background of eight ecological factors. Folia Oecologica, 42(2), 75-88.

Brygadyrenko, V. V. (2015b). Influence of moisture conditions and mineralization of soil solution on structure of litter macrofauna of the deciduous forests of Ukraine steppe zone. Visnyk of Dnipropetrovsk University, Biology, Ecology, 23(1), 50-65.

Brygadyrenko, V. V. (2015c). Parameters of ecological niches of Badister, Licinus and Panagaeus (Coleoptera, Carabidae) species measured against eight ecological factors. Baltic Journal of Coleopterology, 15(2), 137-154.

Brygadyrenko, V. V. (2016). Evaluation of ecological niches of abundant species of Poecilus and Pterostichus (Coleoptera: Carabidae) in forests of the steppe zone of Ukraine. Entomologica Fennica, 27(2), 81-100.

Brygadyrenko, V. V., \& Korolev, O. V. (2015). Morphological polymorphism in an urban population of Pterostichus melanarius (Illiger, 1798) (Coleoptera, Carabidae). Graellsia, 71(1), e025.

Brygadyrenko, V. V., \& Reshetniak, D. Y. (2014a). Morphological variability among populations of Harpalus rufipes (Coleoptera, Carabidae): What is more important - the mean values or statistical peculiarities of distribution in the population? Folia Oecologica, 41(2), 109-133.
Brygadyrenko, V. V., \& Reshetniak, D. Y. (2014b). Trophic preferences of Harpalus rufipes (Coleoptera, Carabidae) with regard to seeds of agricultural crops in conditions of laboratory experiment. Baltic Journal of Coleopterology, 14(2), 179-190.

Brygadyrenko, V. V., \& Reshetniak, D. Y. (2016). Morphometric variability of Clitellocephalus ophoni (Eugregarinida, Gregarinidae) in the intestines of Harpalus rufipes (Coleoptera, Carabidae). Archives of Biological Sciences, 68(3), 587-601.

Czechowski, W. (1981). Carabids (Coleoptera, Carabidae) Warsaw and Mazowia. Memorabilia Zoologica, 34, 119-144.

Dekhtyareva, E. A. (2002). Zoogeograficheskij, biotopicheskij i jekologicheskij analiz pedofauny nasekomyh lesoparkov g. Har'kova [Zoogeographical, biotopical and ecological analysis of the pedofauna of the insects of the forest parks in Kharkov]. The Kharkov Entomological Society Gazette, 10, 123125 (in Russian).

Dekhtyareva, E. A. (2004). Pedofauna vyshhyh komah parkiv m. Harkova [Pedofauna of the highest insects of the Kharkiv parks]. Dokuchaev Agricultural University, Kharkiv (in Ukranian).

Franzen, B. (1992). Untersuchungen zur Käferfauna an ausgewählten Standorten in Köln (Insecta: Coleoptera). Decheniana, 150, 181-216.

Halinouski, N. G., \& Krytskaya, A. M. (2014). An ecological and faunistic review of ground beetles (Coleoptera, Carabidae) in Gomel urbocenosis (the Republic of Belarus). Vestnik Zoologii, 48(6), 521-532.

Hurka, K., \& Jedlickova, Z. (1990). Fauna of carabid beetles (Coleoptera, Carabidae) of Prague. Acta Socientatis Zoologicae Bohemoslovacae, 54(1), 9-17.

Kirichenko, M. B., \& Danylkiv, J. M. (2011). Vydove riznomanittya zhukiv (Coleoptera, Cicindelidae, Carabidae) pryrodoohoronnyh terytorij m. Kyeva [The species diversity of beetles (Coleoptera, Cicindelidae, Carabidae) on the protected areas in the city of Kyiv]. Vestnik Zoologii, 45(5), 411-420 (in Ukrainian).

Kirichenko-Babko, M. B., Kobzar, L. I., Danylkiv, J. M., Łagód, G., \& Franus, M. (2019). Distribution of the carabid species (Coleoptera, Carabidae) in woodlands of the protected and urban areas (north of Ukraine). Vestnik Zoologii, 53(2), 89-106.

Klausnitzer, B. (1990). Jekologija gorodskoj fauny [Ecology of the urban fauna]. Mir, Moscow (in Russian).

Klausnitzer, B. (1983). Faunistisch-Ökologische Untersuchungen über die Laufkäfer (Coleoptera, Carabidae) des Stadtgebietes von Leipzig. Entomologische Nachrichten und Berichte, 27, 241-261.

Komaromi, N. A., Nikolenko, N. Y., \& Puchkov, A. V. (2018). Faunisticheskij sostav zhestkorylyh (Insecta: Coleoptera) gerpetobija urbocenozov g. Kharkova (Ukraina) [The faunistic structure of the beetles (Insecta: Coleoptera) in herpetobios of urbocenosis of Kharkov]. Ukrainian Entomological Journal, $15,3-21$ (in Russian).

Komaromi, N. A., Putchkov, A. V., \& Nazarenko, V. Y. (2019). Osobennosti sezonnyh izmenenij taksonomicheskoj struktury i chislennosti zhukov-dolgonosikov (Coleoptera, Curculionidae) gerpetobija urbocenozov g. Harkova (Ukraina) [Peculiarities of seasonal changes in taxonomic structure and number of weevilbeetles (Coleoptera, Curculionidae) in herpetobios of Kharkiv urbocenoses (Ukraine)]. Ukrainian Entomological Journal, 16, 25-35 (in Russian).

Komlyk, V. O., \& Brygadyrenko, V. V. (2019). Morphological variability of Bembidion minimum (Coleoptera, Carabidae) populations under the influence of natural and anthropogenic factors. Biosystems Diversity, 27(3), 250-269.

Komlyk, V., \& Brygadyrenko, V. (2020). Morphological variability of Bembidion varium (Coleoptera, Carabidae) in gradient of soil salinity. Folia Oecologica, 47, 23-33.

Korolev, O. V., \& Brygadyrenko, V. V. (2014). Influence of individual variation in the trophic spectra of Pterostichus melanarius (Coleoptera, Carabidae) on the adaptation possibilities of its population. Folia Oecologica, 41(1), 34 43.

Kosewska, A., Nietupski, M., \& Damszel, M. (2013). Role of urban forests as a source of diversity of carabids (Coleoptera, Carabidae) in urbanised areas. Baltic Journal of Coleopterology, 13(1), 27-39.

Löbl, I., \& Löbl, D. (Eds.). (2017). Catalogue of Palearctic Coleoptera. Vol. 1. Archostemata - Myxophaga - Adephaga. Brill, Leiden, Boston.

Magura, T., Tóthmérész, B., \& Molnár, T. (2004). Changes in carabid beetle assemblages along an urbanization gradient in the city of Debrecen, Hungary. Landscape Ecology, 19, 747-759.

Magura, T., Tóthmérész, B., \& Molnár, T. (2008). A species-level comparison of occurrence patterns in carabids along an urbanisation gradient. Landscape and Urban Planning, 86(2), 134-140.

Nazarenko, V. Y., \& Petrenko, A. A. (2007). Do vyvchennja fauny zhukiv (Insecta: Coleoptera) Lysoi' gory (m. Kyiv) [To the knowledge of beetle fauna (Insecta: Coleoptera) of Lysa gora (Kyiv)]. The Kharkov Entomological Society Gazette, 15, 43-48 (in Ukrainian).

Nazarenko, V. Y., Putchkov, A. V., \& Komaromi, N. A. (2020). A preliminary inventory of weevil assemblages (Coleoptera, Curculionoidea) in Kharkiv metropolitan area (Ukraine) using pitfall traps. Zoodiversity, 54(2), 123-132.

Niemelä, J., \& Kotze, D. J. (2009). Carabid beetle assemblages along urban to rural gradients: A review. Landscape and Urban Planning, 92(2), 65-71. 
Nikolenko, N. Y. (2018). Ecological and faunistic overview of the carabidofauna (Coleoptera, Carabidae) urbocenoses of Kharkiv. Biology and Valeology, $20,48-54$.

Penev, L., Stoyanov, I., Dedov, I., \& Antonova, V. (2007). Patterns of urbanisation in the City of Sofia as shown by carabid beetles (Coleoptera, Carabidae), ants (Hymenoptera, Formicidae), and terrestrial gastropods (Mollusca, Gastropoda Terrestria). Proceedings of the XIII European Carabidologists Meeting, Blagoevgrad, August 20-24. Sofia-Moscow, Pensoft Publishers. Pp. 483-509.

Puchkov, A. V., Brygadyrenko, V. V., Faly, L. I., \& Komaromi, N. A. (2020). Staphylinids (Coleoptera, Staphylinidae) of Ukrainian metropolises. Biosystems Diversity, 28(1), 41-47.

Putchkov, A. V. (2018). Zhuky-turuny (Coleoptera, Carabidae) transformovanykh tsenoziv Ukrajiny [Ground-beetles (Coleoptera, Carabidae) of transformed cenoses of Ukraine]. I. I. Schmalhausen Institute of Zoology, Kyiv (in Ukrainian).

Putchkov, A. V., \& Aleksandrowicz, O. R. (2020). Zhuzhelicy triby Sphodrini Laporte, 1834 (Coleoptera, Carabidae) fauny Vostochnoj Evropy [Groundbeetles of the tribe Sphodrini (Coleoptera, Carabidae) of East Europe]. Jentomologicheskoe Obozrenie, 99(1), 190-219 (in Russian).

Putchkov, A. V., \& Komaromi, N. A. (2018). Zhuky-karapuziki (Coleoptera, Histeridae) urbocenoziv Kharkova [The clown beetles (Coleoptera, Histeridae) of the urbocenosis of Kharkiv city (Ukraine)]. Biology and Valeology, 20, 54-58 (in Ukrainian).

Putchkov, A. V., Brygadyrenko, V. V., \& Markina, T. Y. (2019). Ground beetles of the tribe Carabini (Coleoptera, Carabidae) in the main megapolises of Ukraine. Vestnik Zoologii, 53(1), 3-12.

Putchkov, A. V., Kirichenko, M. B., \& Uspensky, G. B. (2003). Zhuzhelicy (Coleoptera, Carabidae) urochish ha Lysaja gora v Kieve [Ground beetles (Coleoptera, Carabidae) of Lysa Hora tract in Kyiv]. Vestnik Zoologii, 16, 111-113 (in Russian).

Putchkov, A. V., Markina, T. Y., \& Komaromi, N. A. (2017a). Ecologo-faunistic review of lamellicorn beetles (Coleoptera, Scarabaeoidea) of urbocenozis of Kharkov city (Ukraine). Biosystems Diversity, 25(4), 263-267.

Putchkov, A. V., Markina, T. Y., \& Skavysh, M. (2016). Predvaritel'nyj obzor gerpetobiontnyh zhukov (Soleoptera) parkovyh nasazhdenij g. Harkova (Ukraina)
[Preliminary review of herpetobiont beetles (Coleoptera) of parks of Kharkiv (Ukraine)]. Ukrainian Entomological Journal, 11, 69-76 (in Russian).

Putchkov, O. V., Nikolenko, N., \& Garkusha, I. A. (2017b). Jekologo-faunisticheskij obzor zhuzhelic triby Pterostichini parkovyh urbocenozov Severnoj i Vostochnoj Ukrainy) [Ecologic-faunistical overview on the ground beetles of Pterostichini tribe (Coleoptera, Carabidae) in park urbocenoses of Northern and Eastern Ukraine]. Biology and Valeology, 19, 69-78 (in Russian).

Reshetniak, D. Y., Pakhomov, O. Y., \& Brygadyrenko, V. V. (2017). Possibility of identifying plant components of the diet of Harpalus rufipes (Coleoptera, Carabidae) by visual evaluation. Regulatory Mechanisms in Biosystems, 8(3), 377-383.

Rizun, V. B., \& Diedus, V. I. (2016). Ekologo-biologichni osoblyvosti ugrupovan' zhukiv-turuniv (Coleoptera, Carabidae) Vynnykivs'kogo lisoparku m. L'vova [Ecologic-biological peculiarities of assambles of ground beetles (Coleoptera, Carabidae) of Vynnykivsky urban forest of Lviv]. Scientific notes of the State Natural History Museum, 32, 129-136 (in Ukrainian).

Rizun, V. B., \& Khrapov, D. S. (2001). Do vyvchennja turuniv (Coleoptera, Carabidae) L'vova (Syhivs'kyj ta Vynnykivs'kyj lisoparky) [On the study of ground beetles (Coleoptera, Carabidae) of Lviv (Sykhivsky and Vynnykivsky urban forests)]. Scientific Notes of the State Natural History Museum of NAS of Ukraine, 16, 103-108 (in Ukrainian).

Ryzhaia, A. V. (2005). Osobennosti fauny zhuzhelic (Coleoptera, Carabidae) zelenyh zon mikrorajonov razlichnogo tipa v g. Grodno [Peculiarities of ground beetles fauna (Coleoptera, Carabidae) in the green zones of microdistricts of different type in Grodno]. Vesnik of Yanka Kupala State University of Grodno, 34(2), 142-148 (in Russian).

Šustek, Z. (1987). Changes in body size structure of carabid communities (Coleoptera, Carabidae) along an urbanization gradient. Biologia, 42(2), 145-156.

Šustek, Z. (2012). Changes in carabid communities (Insecta: Coleoptera) along an urbanization gradient in Madrid (Spain). Muzeum Olteniei Craiova. Oltenia. Studii şi comunicări. Śtiinţele Naturii, 28(2), 73-92. 